University of Louisville

ThinkIR: The University of Louisville's Institutional Repository

\title{
$5-2011$
}

\section{Integration of Ag2Ga nanoneedles on batch fabricated micro- grippers for material property measurement.}

\author{
Peng Xu \\ University of Louisville
}

Follow this and additional works at: https://ir.library.louisville.edu/etd

\section{Recommended Citation}

Xu, Peng, "Integration of Ag2Ga nanoneedles on batch fabricated micro-grippers for material property measurement." (2011). Electronic Theses and Dissertations. Paper 1608.

https://doi.org/10.18297/etd/1608

This Master's Thesis is brought to you for free and open access by ThinkIR: The University of Louisville's Institutional Repository. It has been accepted for inclusion in Electronic Theses and Dissertations by an authorized administrator of ThinkIR: The University of Louisville's Institutional Repository. This title appears here courtesy of the author, who has retained all other copyrights. For more information, please contact thinkir@louisville.edu. 


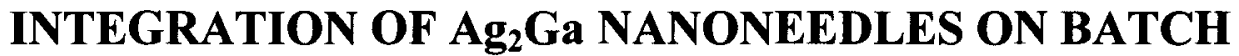 FABRICATED MICRO-GRIPPERS FOR MATERIAL PROPERTY MEASUREMENT
}

\author{
By \\ Peng Xu \\ B.S., Hefei University of Technology, 2009 \\ China
}

\begin{abstract}
A Thesis
Submitted to the Faculty of the

J.B. Speed School of Engineering of the University of Louisville in Partial Fulfillment of the Requirements

for the Degree of

Master of Science

Department of Mechanical Engineering

University of Louisville

Louisville, Kentucky
\end{abstract}

May 2011 
Copyright 2011 by Peng Xu

All rights reserved 
INTEGRATION OF Ag $_{2}$ Ga NANONEEDLES ON BATCH FABRICATED MICRO-GRIPPERS FOR MATERIAL PROPERTY MEASUREMENT

\author{
By \\ Peng Xu \\ B.S., Hefei University of Technology, 2009
}

A Thesis Approved On

April 7th, 2011

By the Following Thesis Committee;

Dr. Balaji Panchapakesan, Thesis Director

Dr. Robert W. Cohn

Dr. Thomas A. Berfield 


\section{DEDICATION}

This thesis is dedicated to my parents

Mr. Qingguo Xu, Mrs. Lunying Lu

and my wife

Mrs. Yan Chen

You have been my greatest source of strength and inspiration. 


\section{ACKNOWLEDGEMENTS}

I would like to thank my advisor Dr. Balaji Panchapakesan for giving me the opportunity to work in the field of micro/nano technology. I would also like to thank Dr. Mehdi Yazdanpanah for his help and guidance in fabrication of the nano-needles. Much of this work was done at the University of Louisville's cleanroom facility and I thank the cleanroom staff for their assistance, training and suggestion.

I also thank my committee, Dr. Robert Cohn and Dr. Thomas Berfield for their valuable suggestions in this work. Finally, I thank the members of the Small Systems Laboratory for their friendship as well as help with the thesis and Jose Rivera at Nauganeedles LLC for help with the fabrication of the nano-needles.

Last but not least, I would like to thank my parents and wife for their love and support. 


\title{
ABSTRACT \\ INTEGRATION OF $\mathrm{Ag}_{2}$ Ga NANONEEDLES ON BATCH FABRICATED MICRO-GRIPPERS FOR MATERIAL PROPERTY MEASUREMENT
}

\author{
Peng Xu
}

March $22^{\text {nd }}, 2011$

In this thesis self-assembled growth of $\mathrm{Ag}_{2} \mathrm{Ga}$ nano-needles on batch fabricated micro-grippers are reported. The room temperature growth of $\mathrm{Ag}_{2} \mathrm{Ga}$ nano-needles have been recently developed as stylus for Atomic Force Microscopy (AFM). Integration of such nano-needles onto batch fabricated micro-grippers can potentially lead to the development of nano-grippers for nano-manipulation of materials, measurement of electrical properties of nanostructures and finally measurement of electrochemical properties of cells.

We explored the growth process of nano-needles on batch fabricated microgrippers. Micro-grippers were first batch fabricated using carbon nanotube/SU8 process at the University of Louisville's clean room facility. Following the gripper fabrication, 20 $\mathrm{nm}$ chromium and $100 \mathrm{~nm}$ silver were selectively deposited onto the arms of the grippers using shadow masking techniques. Each silver coated arm was selectively dipped onto liquid gallium and retracted for the formation of $\mathrm{Ag}_{2} \mathrm{Ga}$ eutectic nano-needles at room 
temperature in ambient environment. The length and diameter of nano-needles can be controlled by the thickness of silver and volume of gallium liquid. Scanning Electron Microscope (SEM) characterization revealed nano-needles of $100-120 \mathrm{~nm}$ in diameter and 7-15 $\mu \mathrm{m}$ long on each gripper. Following the nano-needle growth on the grippers, the grippers were packaged onto a ceramic chip carrier and wire bonded. Following wire bonding, $100 \mathrm{~nm}$ of parylene was coated everywhere besides the surface of gripper/needles to enable electrical insulation in liquid environments, and then the tips of nano-needles are exposed. I-V measurements revealed an open circuit resistance of 120 Giga-ohms showing the versatility of the grippers integrated with the needles as electrodes for measurement of electrical properties of nano-materials and in liquids. Preliminary measurements on a small carbon nanotube film when brought into contact with the needle revealed six to eight orders of magnitude change in the resistance showing that these needles are highly sensitive for electrical property measurement on nano-materials. Finally, the needles were tested inside phosphate buffered saline and performed linear sweep voltammetry to investigate reduction-oxidation reactions on the surface of the nano-needle. 


\section{TABLE OF CONTENTS}

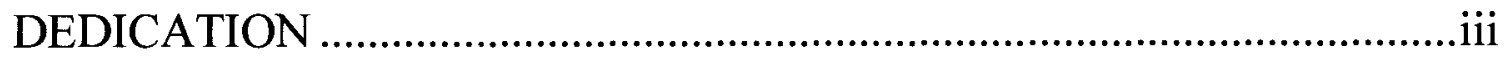

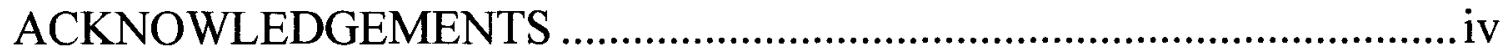

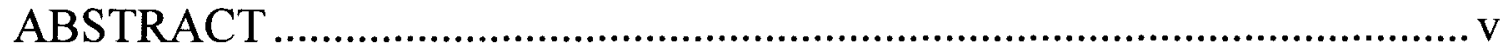

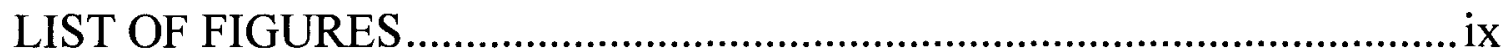

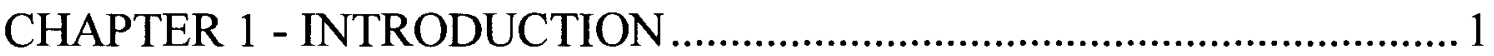

CHAPTER 2 - BACKGROUND AND SIGNIFICANCE ........................... 5

2.1 Nanotube micro-opto-mechanical gripper....................................... 5

2.2 The silver-gallium system ............................................................... 9

2.3 Growth of $\mathrm{Ag}_{2} \mathrm{Ga}$ metallic nano-needles ....................................... 11

CHAPTER 3 - BATCH FABRICATION PROCESS FOR MICRO-

GRIPPERS AND CHARACTERIZATION ........................................... 14

CHAPTER 4 - INTEGRATION OF $\mathrm{Ag}_{2} \mathrm{Ga}$ NANO-NEEDLES ON

MICRO-GRIPPERS AND PACKAGING ............................................... 22

4.1 Nano-needle integration: silicon shadow mask preparation and silver

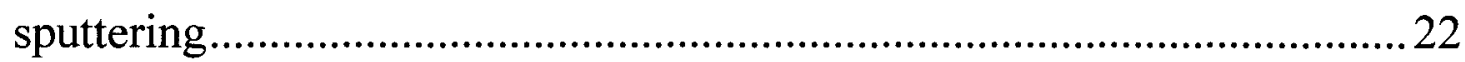


4.2 Nano-needle growth: gallium droplet preparation

4.3 Grow $\mathrm{Ag}_{2} \mathrm{Ga}$ nano-needle on gripper arms....................................2 28

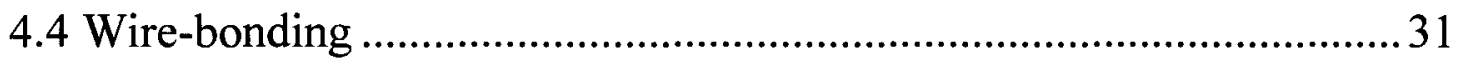

CHAPTER 5 - CHARACTERIZATION OF NANO-NEEDLES AND

MEASUREMENT OF MATERIAL PROPERTIES .................................... 33

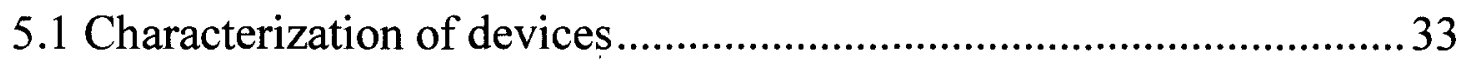

5.2 Measuring electrical property of carbon nanotube film .......................36

5.3 Electrical property measurements in liquids.................................... 41

CHAPTER 6 - CONCLUSION AND FUTURE WORK .......................... 49

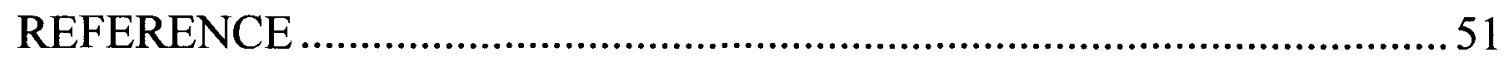

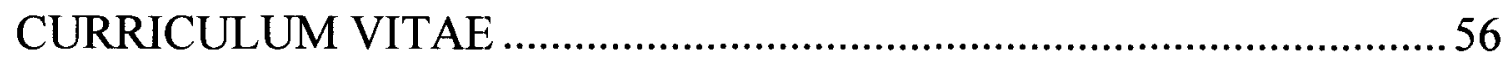




\section{LIST OF FIGURES}

Figure 2.1 SEM images of CNT-MOMS grippers released from the substrate: (a) gripper arrays; (b) magnified square B in figure (a) showing the actuating arms and the supporting arms; (c) magnified square $\mathrm{C}$ in figure (b) showing the crosssectional view of the actuating arms; (d) the magnified square A in figure (a) showing the structure of the gripper tips. [41] ................................................ 6

Figure 2.2 Sequence of a CNT-MOMS gripper attached to a mechanical manipulator to manipulate a micro-polystyrene sphere of $\sim 16 \mu \mathrm{m}$ in diameter: (a) Closed gripper approaching the microsphere; (b) gripper opened under light illumination; (c) gripper closed to grasp the microsphere; (d) the microsphere lifted from the substrate and transferred to destination; (e) microsphere being released to the destination under light illumination on gripper; (f) gripper removed and closed. [41]

Figure 2.3 Displacement as a function of laser power for the micro-grippers demonstrating $2 \mu \mathrm{m}$ displacement at $100 \mathrm{~mW}$ [41].................................... 8

Figure 2.4 The transient response of normalized tip's displacement of the gripper during light switching period: $(\mathbf{\Delta})$ during light "on" period; $(\boldsymbol{\nabla})$ during light "off" period. [41] 
Figure 2.5 Ag-Ga system phase diagram [52]

Figure 2.6 The method for selectively growing individual metallic $\mathrm{Ag}_{2} \mathrm{Ga}$ nano-needles:

(a) schematic images; (b) time sequential SEM images. After dipping the silver coated probe into the gallium (i) the silver dissolves and supersaturates the Ga. After retracting the probe (ii) alloy nano-needles nucleate and grow from the probe tip, with one needle growing past all the others. (iii) The probe is retracted further as the needle grows and finally is removed completely from the gallium when the desired length is achieved $[48,49]$.

Figure 2.7 Selective growth of nano-needles on various substrates and various configurations including: (a) quartz tuning fork; (b) isolated array of three needles grown on a single AFM probe; (c) tungsten tip; (d) array of silicon $10 \mu \mathrm{m}$ diameter micro-pillars; (e) Close up view of a nano-needle grown on one the pillars on (d). $[53,54]$. 13

Figure 3.1 Images of (a) CNTs soot; (b) $0.1 \mathrm{mg} / \mathrm{ml} \mathrm{CNT} \mathrm{solution} \mathrm{before} \mathrm{ultra-sonication;}$ (c) $0.1 \mathrm{mg} / \mathrm{ml}$ CNT solution after 1 hour ultra-sonication; (d) $0.1 \mathrm{~g} / \mathrm{ml} \mathrm{CNT}$ solution after 20 hours ultra-sonication. 14

Figure 3.2 Fabrication process of new type CNT-MOMS grippers. (a) CNTs film is formed on top of MCE filter by vacuum filtration; (b) MCE filter and CNTs film are stick to the silicon wafer; (c) Compressive loading is applied on the top of MCE filter; (d) MCE filter is dissolved, leaving pure CNTs film on the silicon substrate; (e) Deposition a layer of photoresist S1827; (f) S1827 is patterned; (g) CNTs film is etched by oxygen plasma; (h) Deposit a layer of S1827 on the backside; (i) Backside alignment to pattern S1827 on the 
backside; (j) Crystal bonding with a holding wafer; (k) Etching the silicon substrate from backside and separating from the holding wafer ; (l) Dicing tape is stick to the backside and deposition a layer of SU8; (m) SU8 is patterned; (n) Grippers are released by $\mathrm{XeF}_{2}$ dry etching.............................. 16

Figure 3.3 Images of: (a) CNTs strips appear after oxygen plasma etching; (b) SEM image of these CNTs strips ........................................................................ 18

Figure 3.4 SEM pictures of released first design's grippers: (a) grippers with 60 degree angle tip; (b) close-view of 60 degree tip; (c) grippers with 30 degree angle tip; (b) close-view of 30 degree tip. 20

Figure 3.5 Optical micrographs of $2^{\text {nd }}$ generation grippers after isotropic silicon dry etching. 21

Figure 4.1 Silicon shadow mask on top of devices. The shadow mask was used to keep the electrodes from being short during the silver coating. 23

Figure 4.2 Devices are stored in the collection box 24

Figure 4.3 SEM pictures of the second design of gripper with sputter-coated silver: (a) top view; (b) isometric view 25

Figure 4.4 Trench - Shadow Mask Method 26

Figure 4.5 Array of micro-gripper with selective sputter-formed silver by using trench shadow mask method

Figure 4.6 Images of: (a) Gallium droplet on top of silicon micro-pillars; (b) Close up view of suitable and unsuitable size gallium droplet. 27

Figure 4.7 Schematic of the experimental Set-up for growth of nano-needle on the arms of the micro-grippers, inset shows a photograph of the equipment 29 
Figure 4.8 Sequential optical micrographs to grow nano-needles on gripper tips: (a) the tip of right arm is inserted into the gallium droplet, $\mathrm{Ag}$ dissolves and supersaturates the gallium; (b) retract the right tip and $\mathrm{Ag}_{2} \mathrm{Ga}$ nano-needle nucleate and grow; (c) choose another suitable gallium droplet for the left tip; (d) the tip of right arm is inserted into the gallium droplet, $\mathrm{Ag}$ dissolves and supersaturates the gallium; (e) retract the left tip and $\mathrm{Ag}_{2} \mathrm{Ga}$ nano-needle nucleate and grow; (f) close-up view of the nano-needles on the front of nanogripper. 30

Figure 4.9 Image of the package after wire-bonding....... 32

Figure 5.1 SEM images of the first design of nano-grippers: (a) full view; (b) close up view. 33

Figure 5.2 SEM images of nano-needles grown on tips of micro-gripper. 34

Figure 5.3 SEM image of the second design of nano-grippers: (a) full view; (b) close up view 35

Figure 5.4 SEM image of a nano-needle integrated with a micro-gripper tip 36

Figure 5.5 Schematic of the experimental set-up for measuring the CNTs film's surface37 Figure 5.6 Current - Time curve for non-contact mode between nano-needle electrode and CNT film 38

Figure 5.7 Current - Time curve show the huge change between contact mode and noncontact mode of the pair of nano-needle electrodes and the surface of CNTs film. 39 
Figure 5.8 One cycle of current change between non-contact mode and contact mode between the pair of nano-needle electrodes and CNT film surface. There is

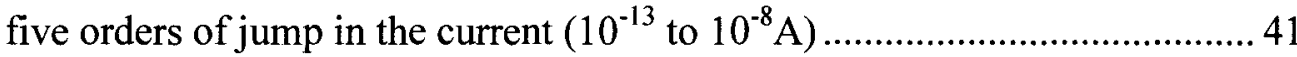

Figure 5.9 Schematic of the experimental set-up for measuring the electrical properties of PBS 42

Figure 5.10 I-V curves when two electrodes in non-contact mode and inside of PBS..... 44

Figure 5.11 Voltage linear change vs. time for ten sweeps' measurement ....................... 45

Figure 5.12 Linear Sweep Voltammetry of PBS with different voltage scanning rates (Scan rates of sweep curves (1-10) correspond to $40-12 \mathrm{mV} / \mathrm{s}$ ) ................... 46

Figure 5.13 A SEM picture of nanoneedle after measurement in PBS .......................... 48 


\section{CHAPTER 1 - INTRODUCTION}

The ability to manipulate atoms, molecules and supra-molecular structures has resulted in an unprecedented understanding of how materials behave at such length scales in the physical, chemical and biological sciences area. Scanning Probe Microscopy (SPM) invented in the early 1980s by Binnig and Rohrer of the IBM Zurich Laboratory has shown the versatility and ease of manipulation and measurement of materials properties of wide variety of materials in air, liquid and vacuum environments [1-9]. However, the single probe tips used in SPMs limit these tools' ability to manipulate objects and measure physical properties; for example, one tip cannot grab an object, and electrical measurements cannot be made without a second contact to structures. It was reported that two or three probe tips are combined for manipulation $[10,11]$ and electrical measurement [12], however it is difficult to connect multiple electrodes to an individual cell for more complex measurements. Two probes in the form of tweezers could overcome these limitations of SPMs and thus might enable new types of fabrication and easy electrical measurements on nanostructures and inside biological cells. MEMS based electrostatic grippers/tweezers, have been fabricated previously on silicon [13-15]. Tungsten deposition and subsequent processing were used to produce tungsten arms 200

$\mu \mathrm{m}$ in length by $2.5 \mu \mathrm{m}$ in width that could be closed by applying a potential of $\sim 150 \mathrm{~V}$ 
and then opened again by reducing $\mathrm{V}$ to zero [13]. The potential difference between the tungsten tweezers arms produces an attractive electrostatic force which can overcome the elastic restoring force of the arms in closing the tweezers. Smaller tweezers with single crystal silicon arms $30 \mu \mathrm{m}$ long by $0.25 \mu \mathrm{m}$ long, which respond at a potential of $45 \mathrm{~V}$, have also been proved with conventional lithography and processing steps [14]. Such micro-tweezers, if removed from the substrate support, could be useful tools for manipulation but are limited by their relatively large size and large actuating voltages for nano-scale work. Electro-thermal grippers/tweezers based on silicon and polymer are also reported [16-22], however they need a high temperature and high driving voltages and these conditions will bring noise for measuring electrical properties of cells. Piezoelectric grippers/tweezers have also been used [23-30]; although they supply a precise actuation technique, they produce small displacements limiting their applications. Pneumatically driven grippers/tweezers, the actuation part is divided from the grippers/tweezers, taking additional complexity in the design and fabrication [31]. Shape memory alloys-based grippers/tweezers $[32,33]$ provide large actuation strokes, however their short lifecycle are not suitable for repeatable measurement.

Recently, several types of systems has been developed at the nano-scale: Kim et al, reported a gripper by using two multiwall carbon nanotubes to attached a glass pipette and showed the measurement of material properties of single nanowires [34]. Similarly, Akita et al. reported carbon nano-tweezers similar to that of Kim and Lieber [35]. However such grippers had to be hand assembled under an optical microscope and they are not compatible with batch fabrication techniques. Cagliani et al. reported a nanogripper for manipulating sub-100nm nanostructures. While impressive, these grippers 
were actuated by electro-thermal actuation, heating the grippers to $300^{\circ} \mathrm{C}$ at two tips and is not suitable for cellular measurements [36]. A nano-gripper employing aligned multiwall carbon nanotube was reported by Jang et al. However high voltage $600 \mathrm{~V}$ for electrostatic actuation was used and these grippers cannot be used in liquids due to the high voltages associated [37]. A type of micro-machined nano-tweezer was reported by Hashiguchi et al., for manipulating small molecules such as DNA [38].

While there have been plenty of grippers reported, some of these grippers have been commercialized for manipulation of materials, devices and systems at the nanoscale. This includes the Zyvex [39] and Femto-tool micro-gripper [40] for manipulation of materials and devices inside an SEM and they are not compatible with biological materials as in measurement of electrical properties in cells since large tips cannot be applied to measure electrochemical properties of cells. Batch fabrication of nano-grippers could open new areas in live measurement of electrical signals in biological materials such as neurons and cells, mechanical and electrical property measurements at the nanoscale, 3-D manipulation using an $\mathrm{X}-\mathrm{Y}-\mathrm{Z}$ nano-manipulator integrated with the nanotweezers.

In this thesis, we explored the growth of $\mathrm{Ag}_{2} \mathrm{Ga}$ nano-needles on previously developed batch fabricated carbon nanotube based photo-mechanically actuated microgrippers. These grippers were ideal platforms for growing nano-needles at the tips of the grippers to act as electrodes for electrical property measurement. Photo-mechanically actuated micro-grippers were developed recently and its utility has been shown for manipulation of small particles such as poly-styrene microspheres [41]. Integration of metallic nano-needles would pave the way for batch fabricated nano-grippers for 
manipulation of single nanotubes, nanowires, cells and other biological materials. This technique also supplies a way to grabbing small nanoparticles while measuring their electrical/electro-chemical properties in air/liquid. Further, the metallic nano-needles could in principle be used as electrodes for measurement of ion-channel activity in cells. 


\section{CHAPTER 2 - BACKGROUND AND SIGNIFICANCE}

\subsection{Nanotube micro-opto-mechanical gripper}

The first batch fabricated nanotube micro-opto-mechanical gripper was first developed by $\mathrm{Lu}$ et al. in 2007 [41]. This type of micro-gripper is based on photomechanical actuation of carbon nanotubes (CNTs) discovered by Ijima et al., [42] inside a scanning electron microscope (SEM). By combining CNTs with a MEMScompatible SU8 polymer, photo-mechanical actuation was enabled in these microsystems. SU8 polymer was used due to its high aspect ratio, mechanical strength, and compatibility with standard micro-fabrication methods. Integration of SU8 polymer as a mechanical and structural element with carbon nanotube has led to development of robust photomechanical actuators and its applications in the development of micro-grippers, micro-cantilevers and micro-mirrors [41, 43, 44]. The advantages of photo-actuation includes the ability to power and control the devices remotely in a non-contact mode, improved signal-to-noise ratio, low electromagnetic interference, and massively parallel manipulation using one light source. 


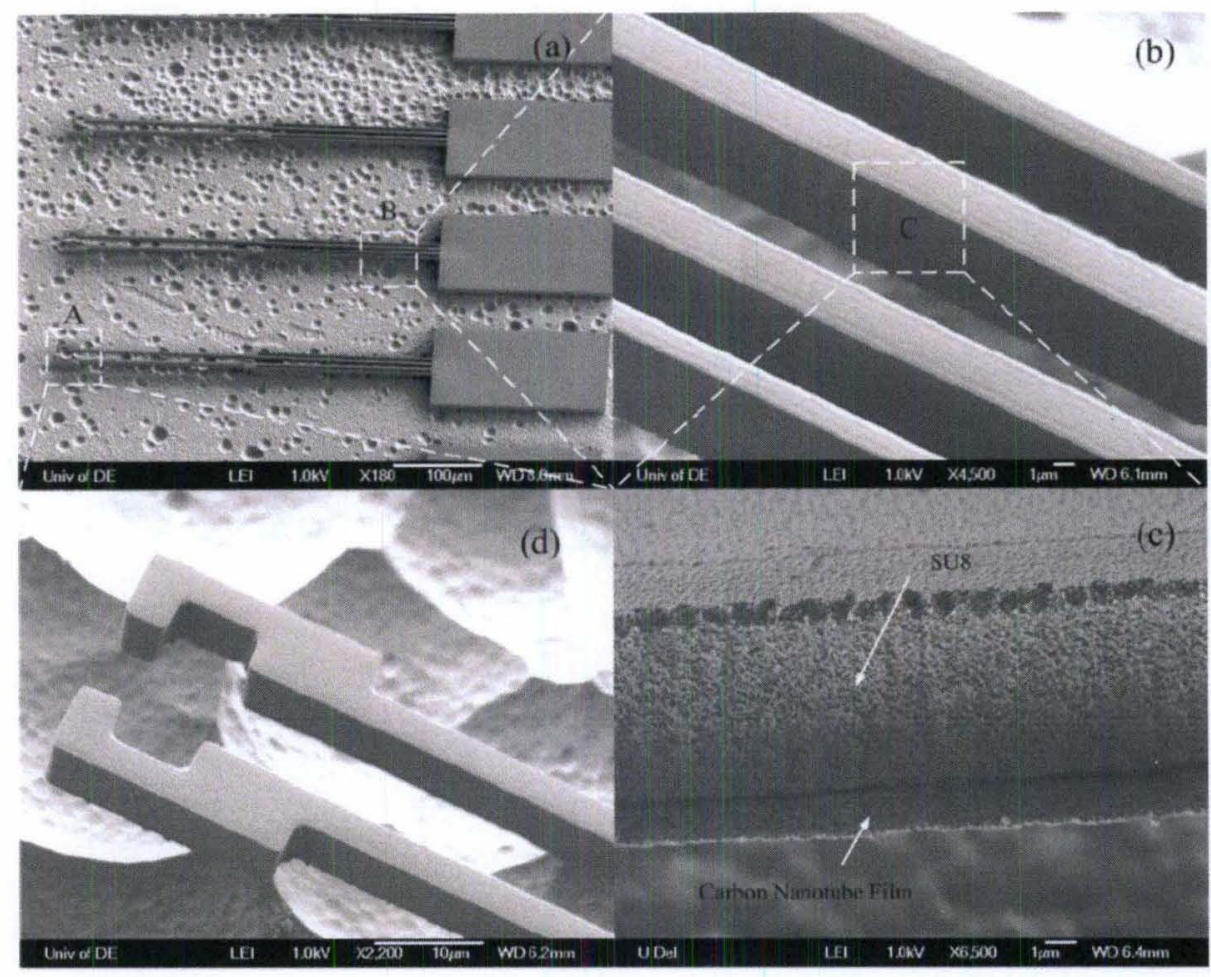

Figure 2.1 SEM images of CNT-MOMS grippers released from the substrate: (a) gripper arrays; (b) magnified square $B$ in figure (a) showing the actuating arms and the supporting arms; (c) magnified square $\mathrm{C}$ in figure (b) showing the crosssectional view of the actuating arms; (d) the magnified square $A$ in figure (a) showing the structure of the gripper tips. [41]

An array of micro-grippers was fabricated as shown in the SEM image of Figure 2.1 (a). Infrared laser is used to operate these grippers simultaneously thereby enabling parallel manipulation. Most MEMS and nanotechnology-based tweezers, which operate on an electrostatic actuation principle and need high power source, are not possible to operate simultaneously. In contrast, optical actuation enables remote powering of devices, remote control, and the ability to provide energy in a non-contact mode. SEM 
micrographs of grippers fabricated using nanotube-film-patterning process is shown in Figure 2.1. These grippers have the dimension of $430 \mu \mathrm{m}$ in length and $20 \mu \mathrm{m}$ in width. Under infrared laser illumination, a lềngth difference between the actuating arm and the supporting arm of gripper enables the actuation. A displacement of $24 \mu \mathrm{m}$ was obtained with infrared laser stimulus of $800 \mathrm{~mW}$, the displacement which is very similar to that achieved with electrically actuated micro-gripper $[20,22,34]$. Sequence SEM images shown in Figure 2.2 involve the micro-manipulation of polystyrene microspheres $(16 \mu \mathrm{m}$ length in diameter) using the nanotube optically actuated micro-gripper. The grippers were mounted on top of the probing arm of a probe station and the polystyrene spheres were subsequently manipulated from one place to another. Figure 2.3 shows the relationship between the displacement of the tip and the laser power. The gripper openings and closing can be performed within a couple of milli-seconds as shown in the transient responses of the gripper in Figure 2.4.

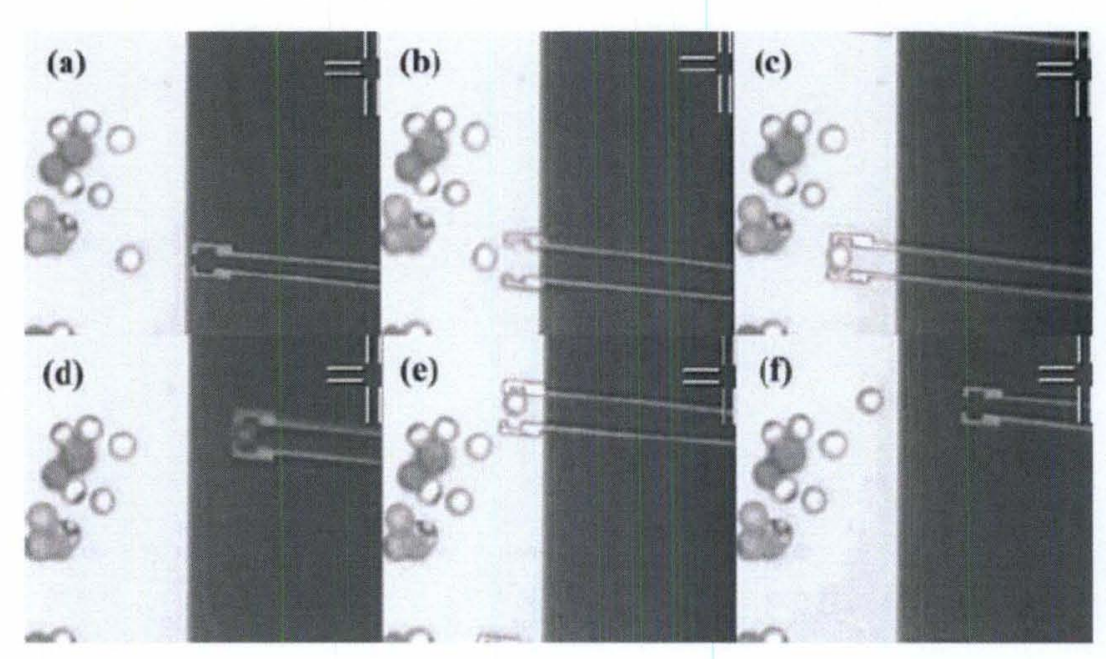

Figure 2.2 Sequence of a CNT-MOMS gripper attached to a mechanical manipulator to manipulate a micro-polystyrene sphere of $\sim 16 \mu \mathrm{m}$ in diameter: (a) 
Closed gripper approaching the microsphere; (b) gripper opened under light illumination; (c) gripper closed to grasp the microsphere; (d) the microsphere lifted from the substrate and transferred to destination; (e) microsphere being released to the destination under light illumination on gripper; (f) gripper removed and closed. [41]

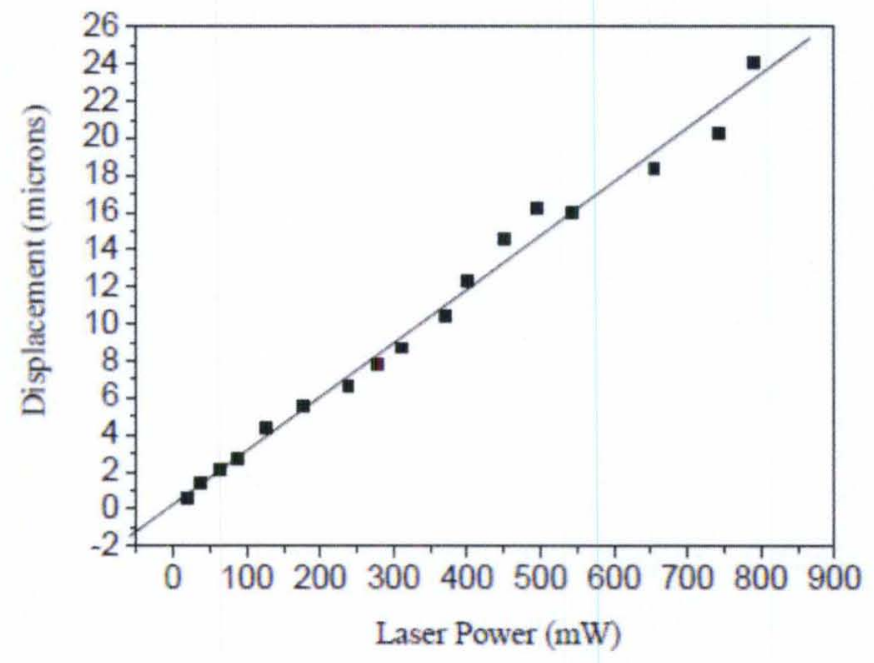

Figure 2.3 Displacement as a function of laser power for the micro-grippers demonstrating $2 \mu \mathrm{m}$ displacement at $100 \mathrm{~mW}$ [41] 


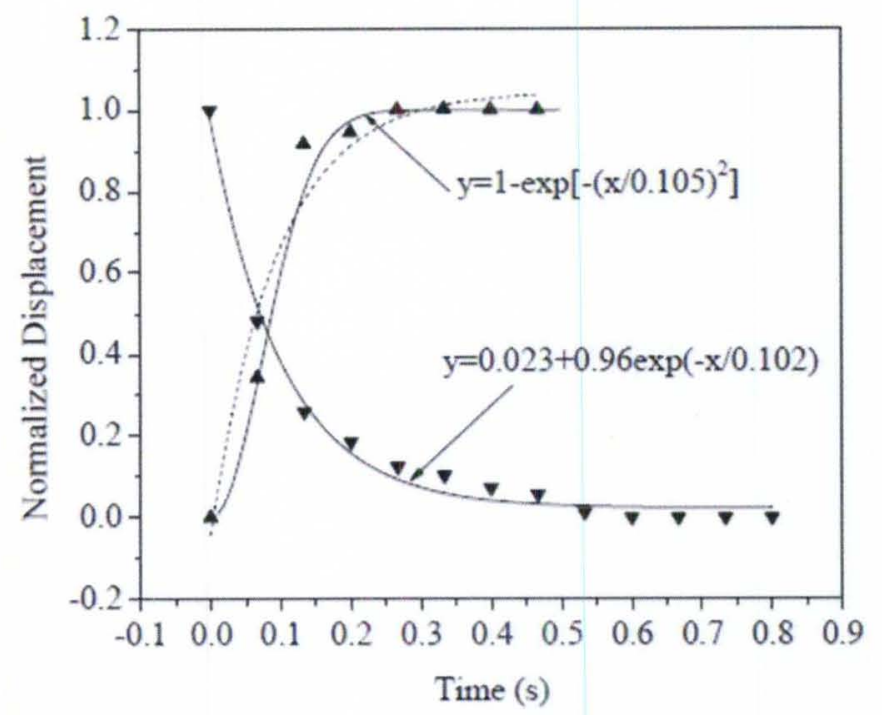

Figure 2.4 The transient response of normalized tip's displacement of the gripper during light switching period: $(\Delta)$ during light "on" period; $(\nabla)$ during light "off" period. [41]

\subsection{The silver-gallium system}

Recently, metallic nano-needles based on $\mathrm{Ag}_{2} \mathrm{Ga}$ intermetallic has been developed with control over direction, diameter, and length at any selected location [45-51]. These needles have been demonstrated to be useful as stylus for atomic force microscopes. The ease of fabrication, growth of the needles from variety of substrates and room temperature processing are quite useful for device development. The phase diagram of the silver-gallium system is presented in Figure 2.5. In the Ag-Ga system phase diagram, the low temperature eutectic point for $\mathrm{Ag}-\mathrm{Ga}$ system is found at the intersection between $26^{\circ} \mathrm{C}$ temperature line and the invariant line at about $98 \% \mathrm{Ga}$ on this phase diagram. 
Figure 2.5 shows that near room temperature the invariant line that is formed between the intermediate phase and the Ga-rich phase, this horizontal line represents the lowest temperature at which a liquid phase for the alloy is found. The $\mathrm{Ag}_{2} \mathrm{Ga}$ intermetallic nano-needle can be formed in ' $\xi$ ' region of the figure. From Figure 2.5 phase diagram of $\mathrm{Ag}-\mathrm{Ga}$ system, it is clear that several invariant reactions occur in the $\mathrm{Ag}-\mathrm{Ga}$ system at low temperature that leads to the formation of $\mathrm{Ag}_{2} \mathrm{Ga}$ intermetallic.

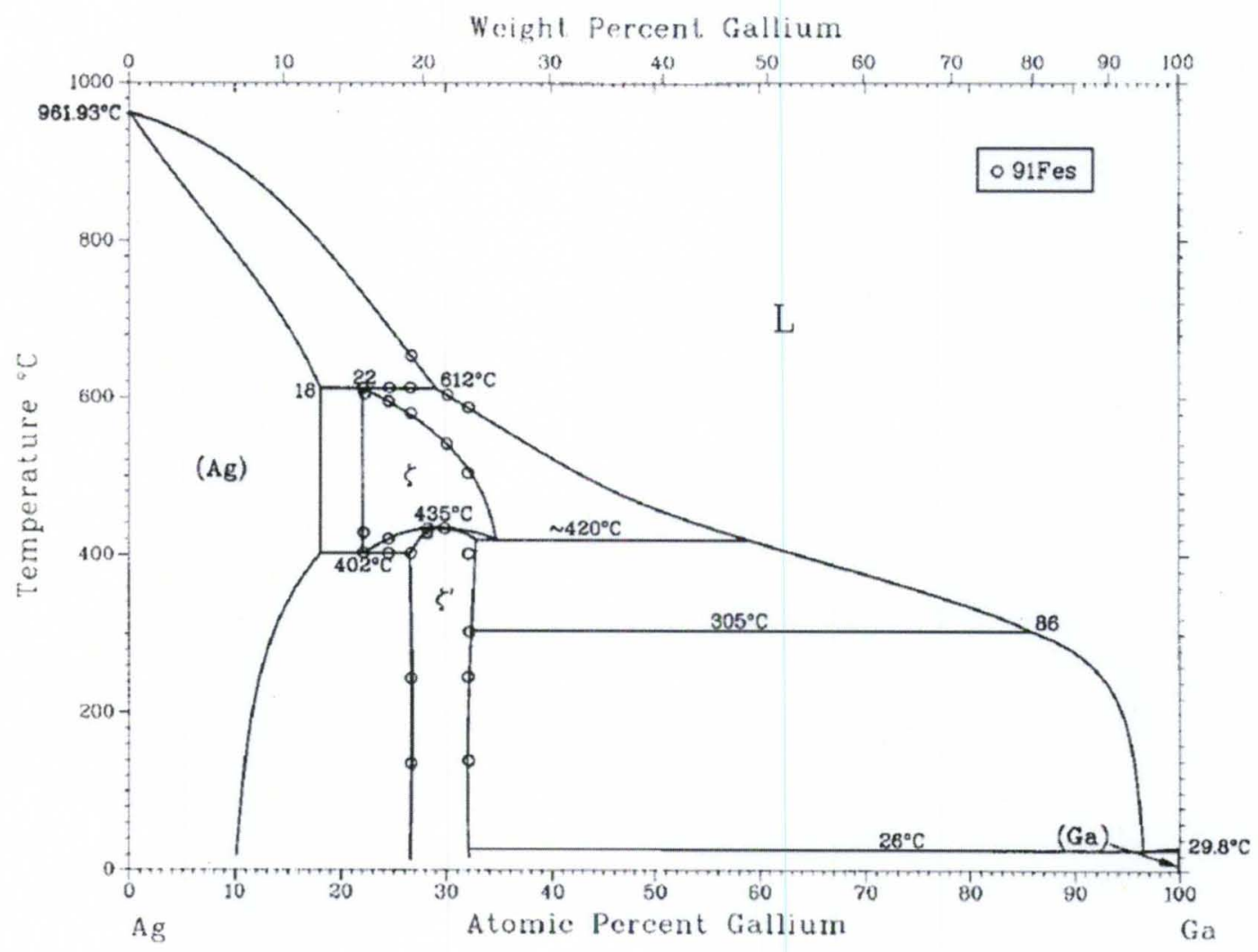

Figure 2.5 Ag-Ga system phase diagram [52] 


\subsection{Growth of $\mathrm{Ag}_{2} \mathrm{Ga}$ metallic nano-needles}

By bringing liquid gallium in contact with sputter deposited silver on a tip and enabling slow retraction, one can preferentially enable growth of silver-gallium phases as nano-needles in the direction of the retraction of the tip $[45,47,48]$. These nano-needles form easily in liquid gallium at room temperature (melting point of gallium is $29.7646^{\circ} \mathrm{C}$ ).

Figure 2.6 illustrates how $\mathrm{Ag}_{2} \mathrm{Ga}$ nano-needles are induced to grow on $\mathrm{AFM}$ probes. First, the probe is sputter-coated with chromium and silver to a typical thickness of $20 \mathrm{~nm}$ and $100 \mathrm{~nm}$ respectively. Then, the probe is dipped into molten gallium at room temperature (Figure 2.6 (a-Left)). The silver layer dissolves and at the same time the silver and gallium crystallize within the meniscus (Figure 2.6 (a-Middle)), forming a freestanding nano-needle when the cantilever is pulled away from the droplet (Figure 2.6 (a-Right)). This process is generally described as a type of directed self-assembly. The time sequential SEM images of growing a needle $(100 \mathrm{~nm}$ in diameter and $60 \mu \mathrm{m}$ in length) inside the chamber of SEM is shown in Figure 2.6 (b). By using a nanomanipulator, an operator can manually control the position of the AFM probe. The nanoneedle growth process also has been performed repeatedly in ambient air under high magnification optical microscope. The time required for nano-needles to form ranges from a few seconds to a few minutes. 

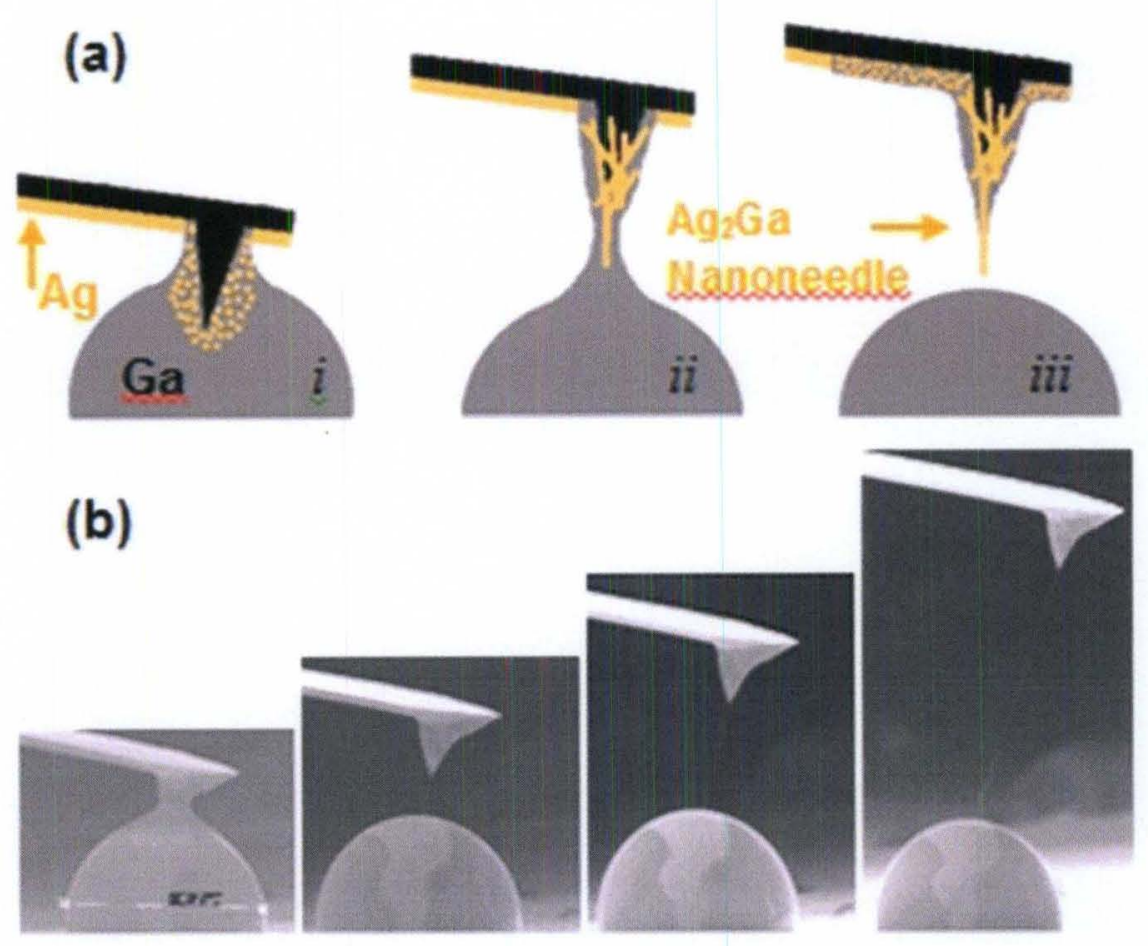

Figure 2.6 The method for selectively growing individual metallic $\mathrm{Ag}_{2} \mathrm{Ga}$ nanoneedles: (a) schematic images; (b) time sequential SEM images. After dipping the silver coated probe into the gallium (i) the silver dissolves and supersaturates the Ga. After retracting the probe (ii) alloy nano-needles nucleate and grow from the probe tip, with one needle growing past all the others. (iii) The probe is retracted further as the needle grows and finally is removed completely from the gallium when the desired length is achieved $[48,49]$.

The nano-needles have been fabricated onto substrates of various compositions and geometries, including quartz resonator tuning forks as shown in (Figure 2.7 (a)), tipped and tipless cantilevers (Figure 2.7 (b)), and tungsten probes (Figure 2.7 (c)), using the same method demonstrate in Figure 2.6. It should be noted that sharp point is not 
necessary for growing nano-needles and freestanding nano-needles can be grown on any silver coated substrate.

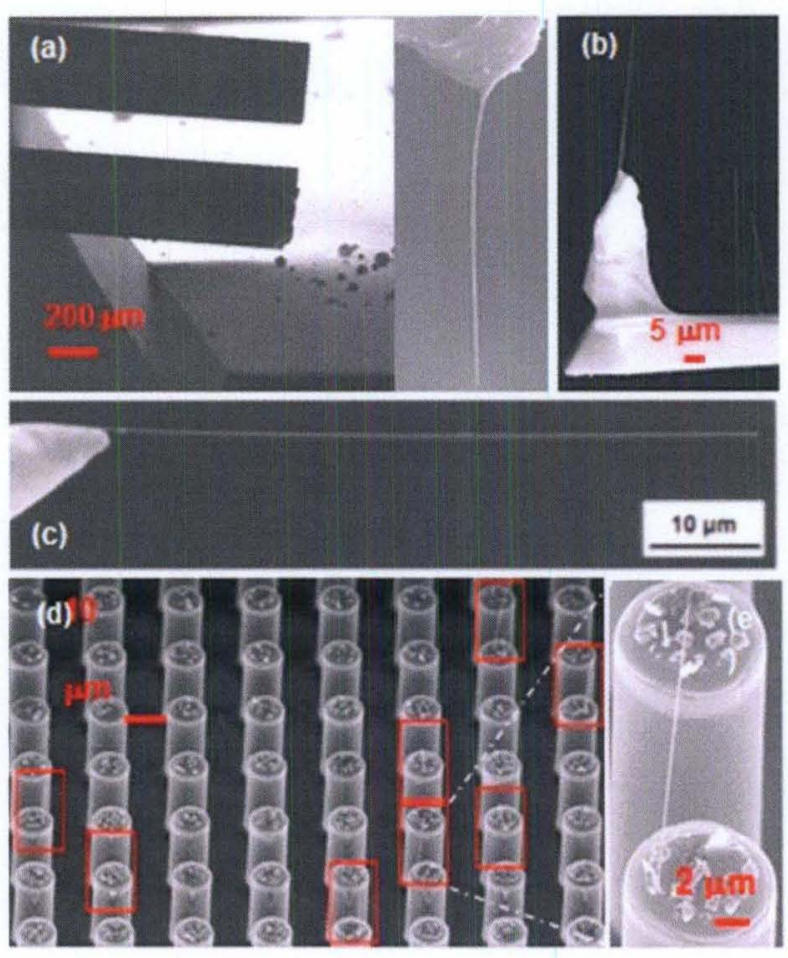

Figure 2.7 Selective growth of nano-needles on various substrates and various configurations including: (a) quartz tuning fork; (b) isolated array of three needles grown on a single AFM probe; (c) tungsten tip; (d) array of silicon 10 $\mu \mathrm{m}$ diameter micro-pillars; (e) Close up view of a nano-needle grown on one the pillars on (d). $[53,54]$ 


\section{CHAPTER 3 - BATCH FABRICATION PROCESS FOR MICRO- GRIPPERS AND CHARACTERIZATION}

The first step to make nano-needle electrodes is to batch fabricate the nanotube micro-grippers by using the previously developed process consisting of carbon nanotube film formation/film transfer/film patterning/structure release steps [43].

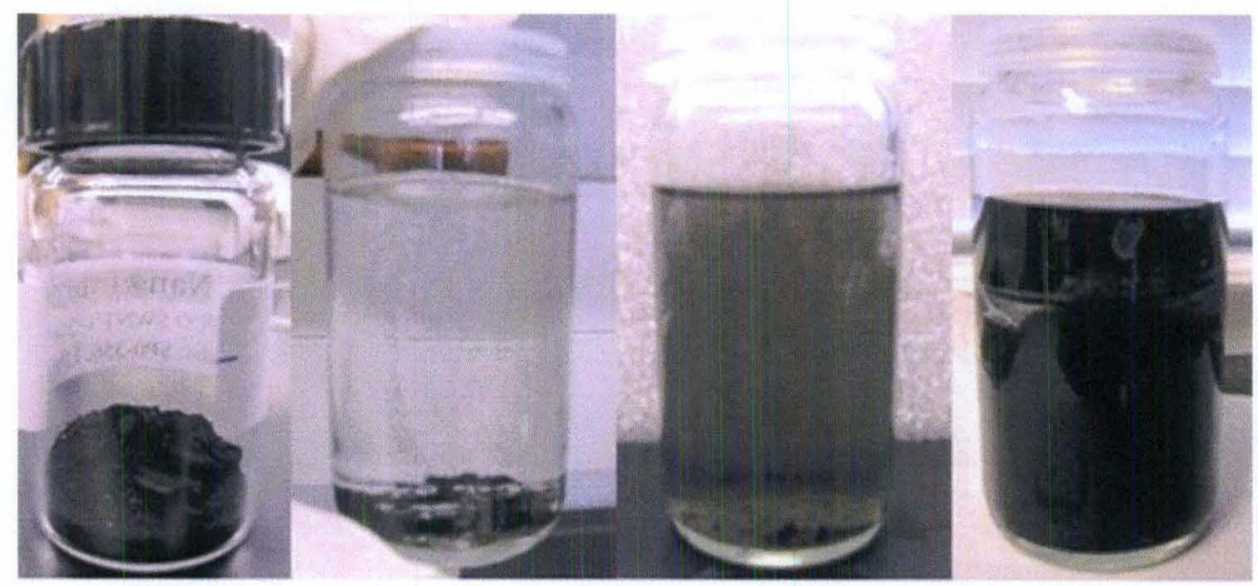

(a)

(b)

(c)

(d)

Figure 3.1 Images of (a) CNTs soot; (b) $0.1 \mathrm{mg} / \mathrm{ml}$ CNT solution before ultrasonication; (c) $0.1 \mathrm{mg} / \mathrm{ml}$ CNT solution after 1 hour ultra-sonication; (d) $0.1 \mathrm{~g} / \mathrm{ml}$ CNT solution after 20 hours ultra-sonication. 
A one-side polished 4" silicon wafer was used as the substrates for the fabrication of the grippers. $10 \mathrm{mg}$ commercial $95 \%$ super purity single wall carbon nanotubes from NanoIntegris was dispersed uniformly into $100 \mathrm{~mL}$ isopropyl alcohol by ultra-sonication $20 \mathrm{hrs}$ to make a concentration of $0.1 \mathrm{mg} / \mathrm{mL}$ solution (Figure 3.1 ). Then isopropyl alcohol was added to dilute the concentration of solution to $0.004 \mathrm{mg} / \mathrm{mL}$ and placed in an ultra-sonication bath again for $20 \mathrm{hrs}$ to make more uniform solution. Highly entangled SWCNTs are separated into individual or small bundle SWCNTs. Then the single wall carbon nanotube solution was filtered using a vacuum filtration technique to produce films of carbon nanotubes on top of a MCE filter as shown in Figure 3.2 (a). The carbon nanotube film on MCE filter is then transferred on to a silicon substrate by compressive loading as shown in Figure $3.2(\mathrm{~b}, \mathrm{c})$. After compressive loading, the nanotube films adhered to the silicon substrate with enough adhesion strength for further processing. The MCE filter is then removed by multi vapor baths of acetone, and then it is rinsed in DI water to leave carbon nanotube film on top of the Si substrate (Figure 3.2 (d)). The thickness of CNT films can be changed by controlling the concentration and the volume of the carbon nanotube solution during vacuum filtration steps. Moderate temperate $\left(75^{\circ} \mathrm{C}\right)$ annealing was performed for 20 minutes to form intimate contact between carbon nanotubes and substrate. Through these processes, uniform carbon nanotube films of desired thickness were readily produced on silicon wafer. Carbon nanotube films that were less than $300 \mathrm{~nm}$ started showing a high degree of transparency. By varying the film thickness and the transparency one can tune the photo-mechanical actuation properties in carbon nanotubes. 
(a)

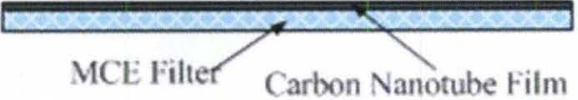

(b)

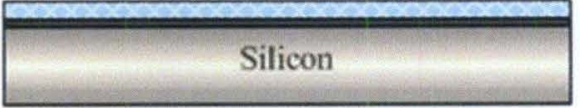

(c)

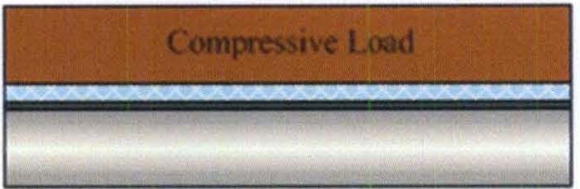

(d)

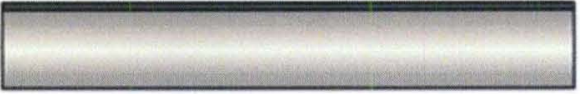

(c)

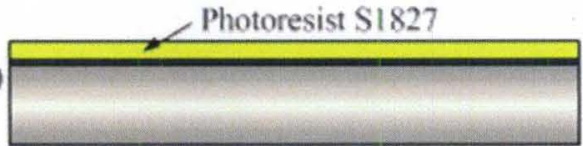

(f)

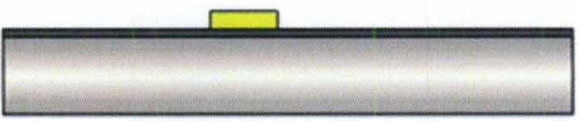

(g)

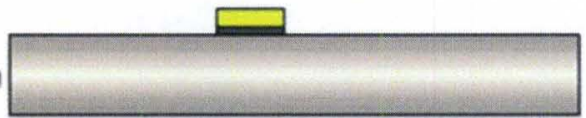

(h)

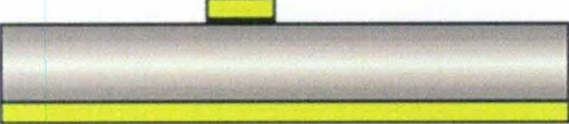

(i)

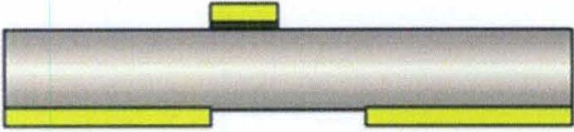

(i)

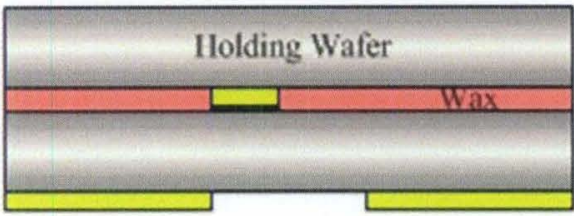

(k)
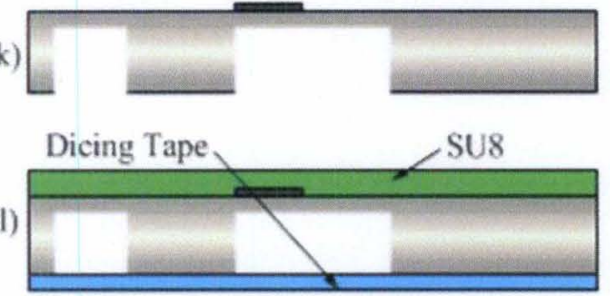

$(\mathrm{m})$

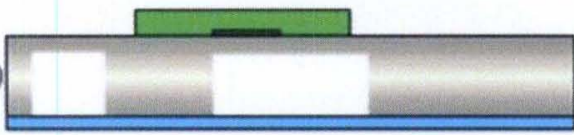

(n)

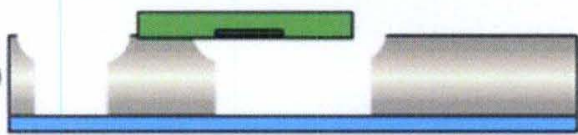

Figure 3.2 Fabrication process of new type CNT-MOMS grippers. (a) CNTs film is formed on top of MCE filter by vacuum filtration; (b) MCE filter and CNTs film are stick to the silicon wafer; (c) Compressive loading is applied on the top of MCE filter; (d) MCE filter is dissolved, leaving pure CNTs film on the silicon substrate; (e) Deposition a layer of photoresist S1827; (f) S1827 is patterned; (g) CNTs film is etched by oxygen plasma; (h) Deposit a layer of S1827 on the backside; (i) Backside alignment to pattern S1827 on the backside; (j) Crystal bonding with a holding wafer; (k) Etching the silicon substrate from backside and separating from the holding wafer ; (I) Dicing tape is stick to the backside and deposition a layer of SU8; (m) SU8 is patterned; (n) Grippers are released by $\mathrm{XeF}_{2}$ dry etching. 
Following nanotube film formation, Deep Reactive Ion Etching (DRIE) was used to pattern the carbon nanotube film by oxygen plasma (Figure $3.2(\mathrm{~g})$ ). Since oxygen plasma also attacks photoresist, the photoresist etch mask needs to be thick enough to sustain continuous oxygen plasma etching. Initial characterization of the etching speed of CNTs and Shipley S1827 (2.7 $\mu \mathrm{m}$ thickness) under different conditions showed that S1827 can be used as CNTs' etch mask only when CNTs film's thickness is smaller than $3.6 \mu \mathrm{m}$ (Table 3.1), also etching ratio 1:0.75 can be reached when the wafer is left for two days after hard bake.

Table 3.1 Etching speed of CNTs and S1827 in different conditions

\begin{tabular}{|c|c|c|c|}
\hline & $\begin{array}{c}\text { Non-hard bake } \\
\text { of S1827 }\end{array}$ & $\begin{array}{c}\text { Hard bake of } \\
\text { S1827 }\end{array}$ & $\begin{array}{c}\text { Leave two days } \\
\text { after hard bake }\end{array}$ \\
\hline Etching speed of CNT & $8 \mathrm{~nm} / \mathrm{s}$ & $8 \mathrm{~nm} / \mathrm{s}$ & $8 \mathrm{~nm} / \mathrm{s}$ \\
\hline Etching speed of S1827 & $40 \mathrm{~nm} / \mathrm{s}$ & $20 \mathrm{~nm} / \mathrm{s}$ & $6 \mathrm{~nm} / \mathrm{s}$ \\
\hline Selectivity ratio & $1: 5$ & $1: 2.5$ & $1: 0.75$ \\
\hline
\end{tabular}

Oxygen plasma has been widely used to remove carbon-based organic materials. It forms volatile $\mathrm{CO}, \mathrm{CO}_{2}$, and $\mathrm{H}_{2} \mathrm{O}$ which can be pumped out from the system during plasma etching process [55]. At ICP power $200 \mathrm{~W}$, bias $\sim 100 \mathrm{~W}, 0.1$ mtorr pressure and $\mathrm{O}_{2}$ flow rate $50 \mathrm{sccm}$, an etch rate of CNT film at $\sim 6 \mathrm{~nm} / \mathrm{s}$ was achieved, showing the fast etching of carbon nanotubes in strong $\mathrm{O}_{2}$ plasma and low pressure. Sharp edges of 
CNT can be seen after etching (Figure 3.3 (a)) and CNT film is characterized by SEM (Figure $3.3(b))$.

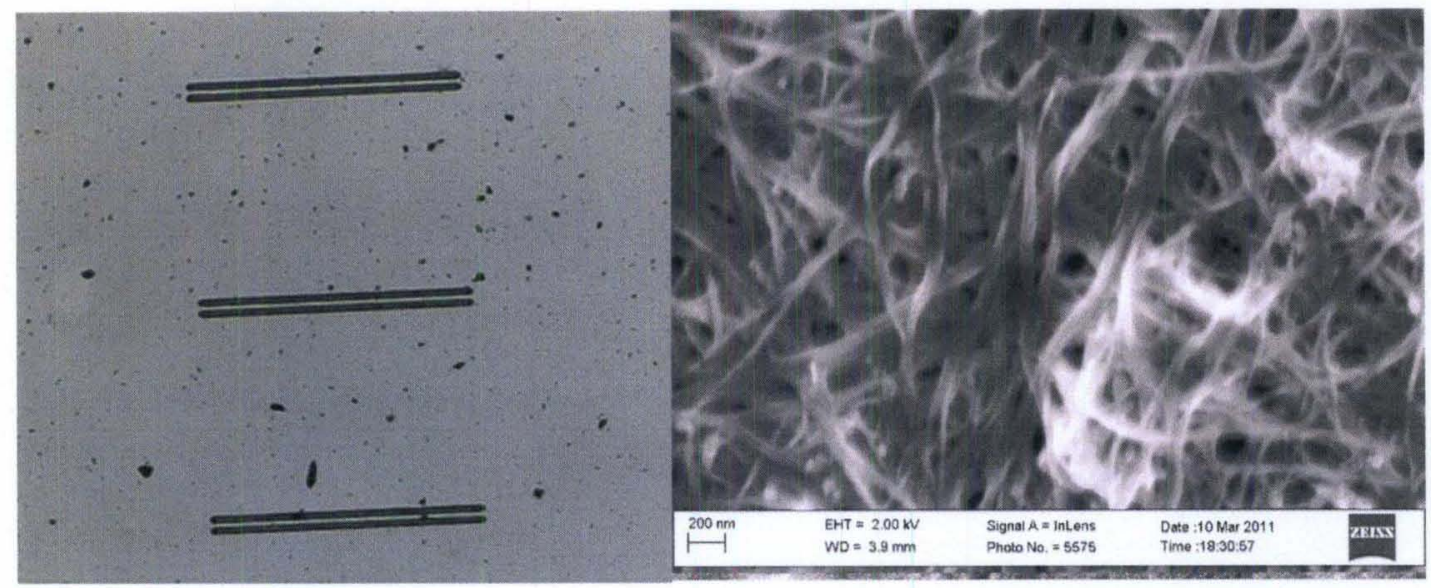

Figure 3.3 Images of: (a) CNTs strips appear after oxygen plasma etching; (b) SEM image of these CNTs strips

Following oxygen plasma etching, a layer of S1827 is deposited at the backside surface (Figure $3.2(\mathrm{~h})$ ) and patterned by backside alignment photolithography (Figure 3.2 (i)). Then deep reactive ion etching was used to etch almost through the silicon substrate from backside. Another silicon wafer is bonded using a wax as shown in Figure 3.2 (j) before back side etching in an ICP top provide mechanical rigidity to the silicon substrate and to avoid cracking. Back side bulk etching of silicon is achieved by monitoring the time of etching. After three and half hours, one can achieve an etching of $\sim 450 \mu \mathrm{m}$ deep to leave behind $50 \mu \mathrm{m}$ silicon layer for later surface fabrication process. The thickness of the remaining silicon wafer was measured using a profilometer (Veeco Dektak). 
Following the silicon backside etching, the bonded wafers are immersed into acetone overnight to dissolve wax to enable the release of the wafers. Further, this immersion in acetone overnight helps in stripping the etching mask S1827 completely to leave a clean surface (Figure $3.2(\mathrm{k})$ ). Then the wafer is gently washed with acetone, methanol, and isopropyl alcohol to remove any residue of photoresists and remove any other impurities from photolithography and etching steps. Finally, an annealing step is performed at 75 degree $\mathrm{C}$ for 20 minutes. The next step is to make sure the thin wafers have enough rigidity and to avoid cracking blue dicing tape is pasted to the bottom of the wafer and SU8 is spun to protect the wafer in case of crack (Figure 3.2 (1)).

Finally, third photolithography is performed to pattern SU-8 layer on top of the carbon nanotube gripper (Figure $3.2(\mathrm{~m})$ ). Then the gripper structures are released from the silicon substrate by xenon difluoride $\left(\mathrm{XeF}_{2}\right)$ isotropic silicon etching (Figure $3.2(\mathrm{n})$ ). High $\mathrm{XeF}_{2}$ pressure and longer circle time can increase the etching speed [56]. The average etch rate $2.1 \mu \mathrm{m} / \mathrm{min}$ can be achieved using cycle time of $120 \mathrm{~s}$ and $\mathrm{XeF}_{2}$ pressure 5.5 torr. Etching process will stop when the wafer is etched through.

We investigated two designs of grippers for growth of the nano-needle. Since $\mathrm{Ag}_{2} \mathrm{Ga}$ nano-needles are formed on the tips of the gripper, their growth direction is determined by the pulling direction and the tip structure. The grippers that were originally conceived had thick and flat tips for holding micro-meter scale objects and it was difficult to grow needles on such surfaces. 


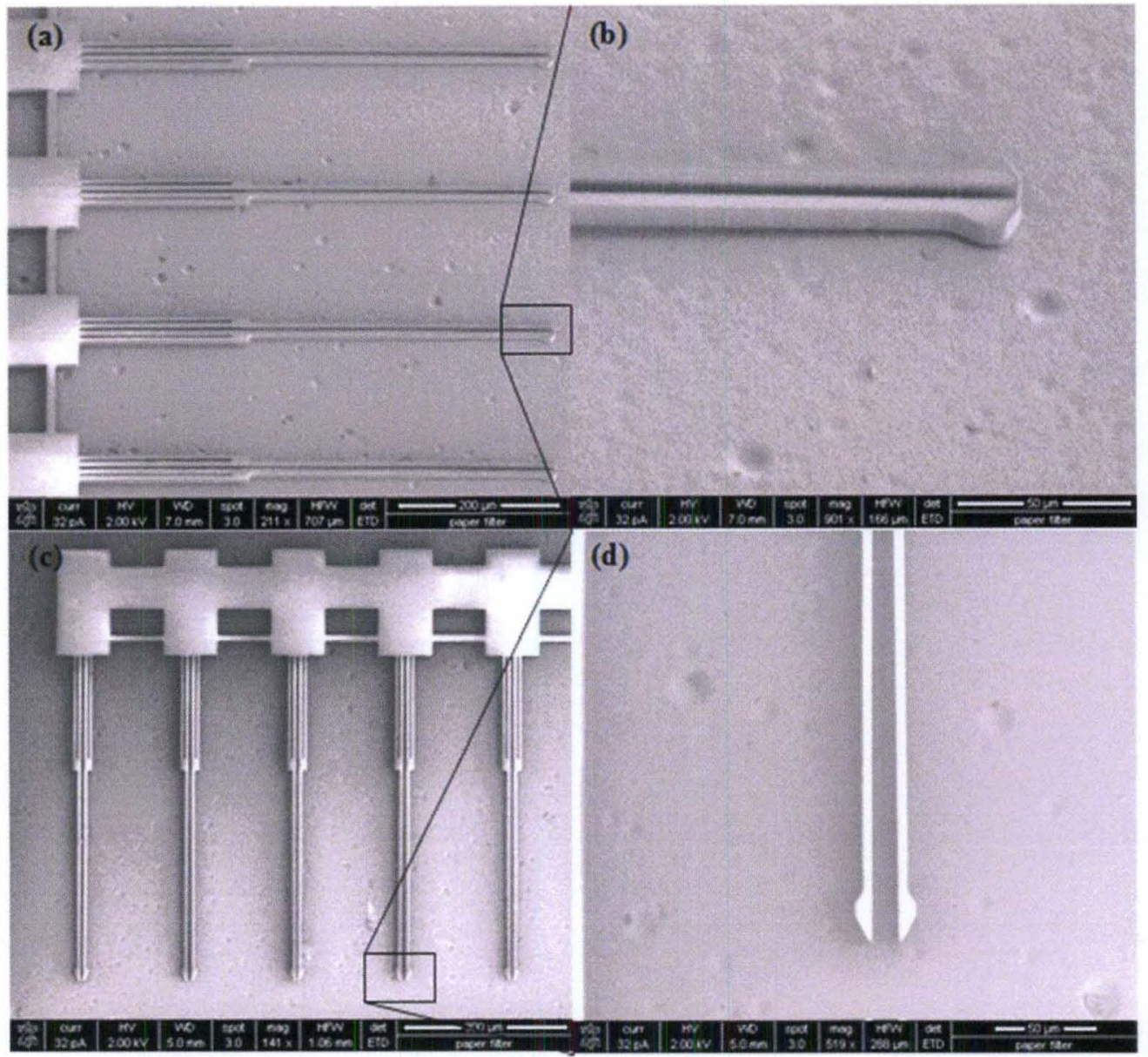

Figure 3.4 SEM pictures of released first design's grippers: (a) grippers with 60 degree angle tip; (b) close-view of 60 degree tip; (c) grippers with 30 degree angle tip; (b) close-view of $\mathbf{3 0}$ degree tip.

The first design we investigated was from carbon nanotube micro-optomechanical grippers by Lu et al. [41]. We modified this design by making the end of the tips sharp with different angles $\left(30^{\circ}, 45^{\circ}\right.$ and $\left.60^{\circ}\right)$ (Figure 3.4). After initial nano-needle growth evaluation, we found that this type of grippers had drawbacks in the integration process with nano-needle. Firstly, the distance between two tips was $4 \mu \mathrm{m}$, which limits the working space making the arms of the grippers too close to each other and made it 
difficult to grow the second needle. Secondly, two arms of the gripper are also too close (4 $\mu \mathrm{m})$, which causes some problem to selectively sputter silver to make two mutually electric-insulated arms. Although the first design has problems with integration, we were able to demonstrate that $\mathrm{Ag}_{2} \mathrm{Ga}$ nano-needle can be integrated with theses SU8 grippers.

Our experience with the first design in the nano-needle fabrication led us to make changes in the second design (Figure 3.5): 1) The distance between two tips of the grippers were increased to $10 \mu \mathrm{m} ; 2$ ) increased the distance between the ends of arms to $150 \mu \mathrm{m}$, so shadow masking could be used easily for sputtering silver onto the arms and also achieve two mutually electric-insulated arms; 3) The area of the gripper base is increased to $3 \mathrm{~mm} \times 4 \mathrm{~mm}$, to enable enough space for wire bonding and integration with the chip.

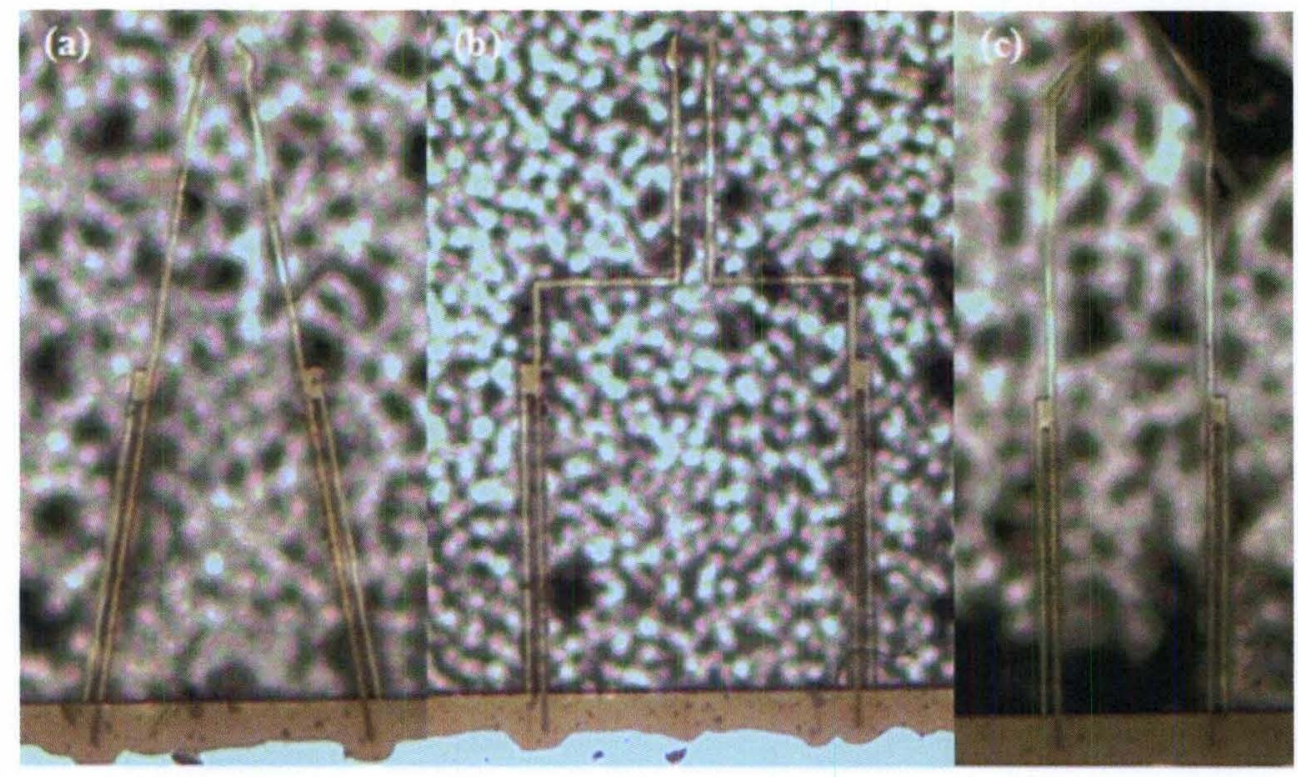

Figure 3.5 Optical micrographs of $2^{\text {nd }}$ generation grippers after isotropic silicon dry etching. 


\section{CHAPTER 4 - INTEGRATION OF Ag,Ga NANO-NEEDLES ON MICRO-GRIPPERS AND PACKAGING}

\subsection{Nano-needle integration: silicon shadow mask preparation and silver sputtering}

In order to probe materials using nano-needles, two electrically conductive needles are required that are completely electrically isolated from one another. The growth process of nano-needles makes this difficult as a thick layer of silver is required before the growth process in liquid gallium. This results in shorting of the grippers and therefore is of no subsequent utility for electrical measurements. In order to overcome this limitation, we have developed shadow masking technique in which an entire shadow mask is prepared on a silicon wafer and subsequently aligned with the wafer containing the devices before chromium and silver deposition in sputtering chamber. Figure 4.1 presents the optical micro-graph of a silicon shadow mask that is aligned on top of the device wafers. 


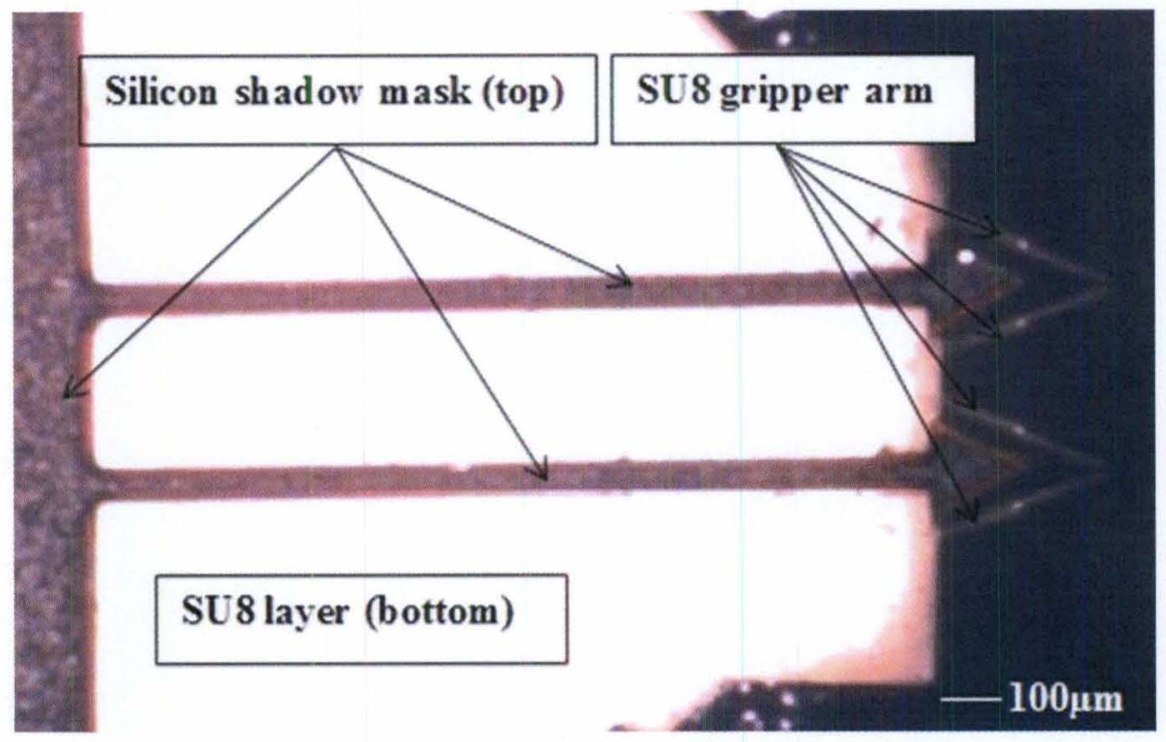

Figure 4.1 Silicon shadow mask on top of devices. The shadow mask was used to keep the electrodes from being short during the silver coating.

The preparation of the shadow mask starts with a lithography process where SPR 220-7 photoresist ( $7 \mu \mathrm{m}$ thickness) is applied to the surface of the wafer followed by lithography to pattern the silicon before etching. The photoresists serves as etch resistive mask, on the 4" silicon wafer. The wafers are then etched using DRIE to through the silicon in pre-patterned layers and define the shadow mask. The silicon shadow mask is then placed on top of the wafer containing the devices and aligned using optical microscope. Pre-designed alignment marks both on the mask and on the wafer enables precise alignment of the wafer as shown in (Figure 4.1). Following alignment, the wafers are anchored together by vacuum tape. After these steps, $20 \mathrm{~nm}$ chromium and $100 \mathrm{~nm}$ silver films are sputter-formed to cover the grippers' wafer through the shadow mask in sputtering machine. 
Then the shadow mask is removed from the grippers' wafer, and bases with grippers are picked one by one to the collection box (Figure 4.2), these devices will be stored in vacuum environment, to eliminate oxidation before nano-needle growth.

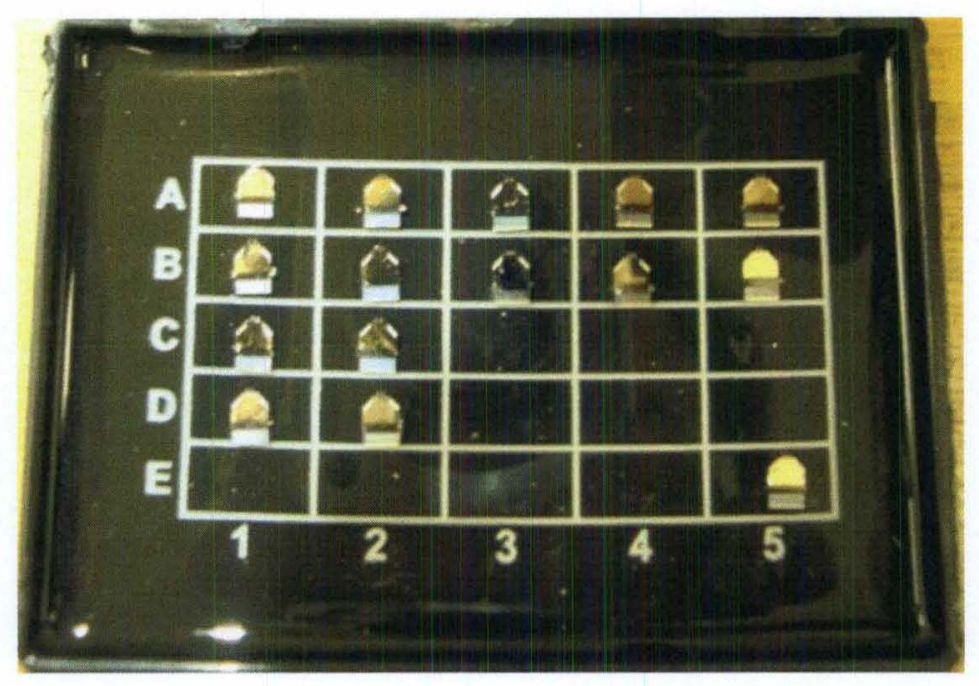

Figure 4.2 Devices are stored in the collection box

By using this shadow masking technique, one can successfully deposit silver on the gripper arms without electrically shorting the devices (Figure 4.3).

We also developed a second process to make grippers with two mutually electricinsulated arms, which is faster to align the wafers than the first shadow mask method. Since in the first shadow mask method, we need to align a $100 \mu \mathrm{m}$ wide barrier to a 150 $\mu \mathrm{m}$ wide center space, it led to considerable time and difficulty of alignment under an optical microscope. Compared with the first shadow mask method, the second method, which is called trench - shadow mask, is much easier to align the wafers without the use of an optical microscope. 
In the trench - shadow mask method, we follow the same fabrication procedure in Figure 3.2 except for changing the mask design for backside silicon etching (Figure 3.2 (i)) and SU8 layer pattern (Figure $3.2(\mathrm{k})$ ) to make a $100 \mu \mathrm{m}$ wide and $2 \mathrm{~mm}$ long trench in the base between two arms of gripper shown in Figure 4.4. After $\mathrm{XeF}_{2}$ etching process, whole wafer with devices is coated with $300 \mathrm{~nm}$ Parylene which is as an insulator layer inside of Parylene Deposition System (PDS 2010 Labcoter 2).

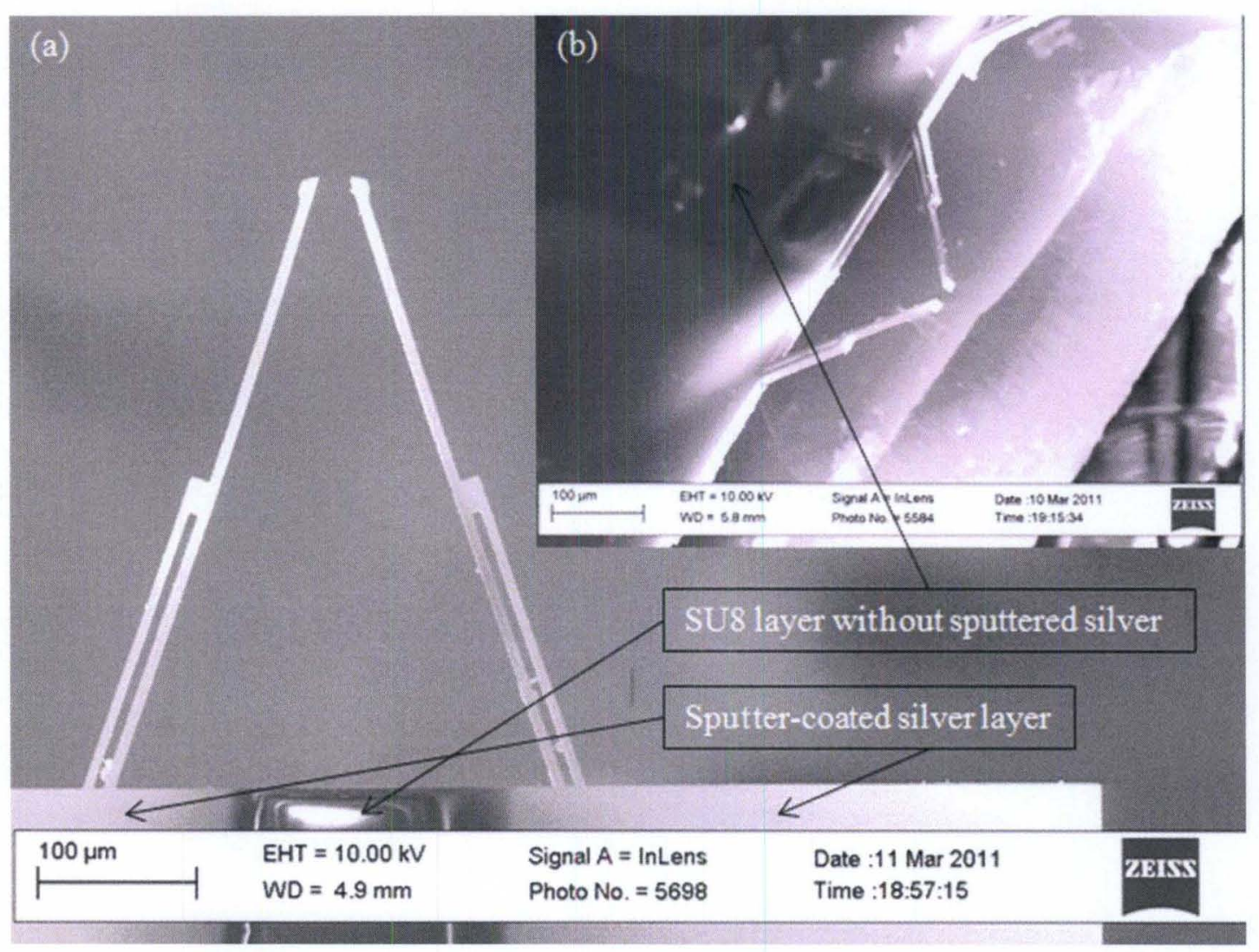

Figure 4.3 SEM pictures of the second design of gripper with sputter-coated silver: (a) top view; (b) isometric view 
Following parylene coating, the silicon shadow mask is aligned on top of the device wafers by eye shown in Figure 4.4 and then bonded together to sputter $20 \mathrm{~nm}$ chromium and $100 \mathrm{~nm}$ silver before needle growth. This alignment step does not require an optical microscope.

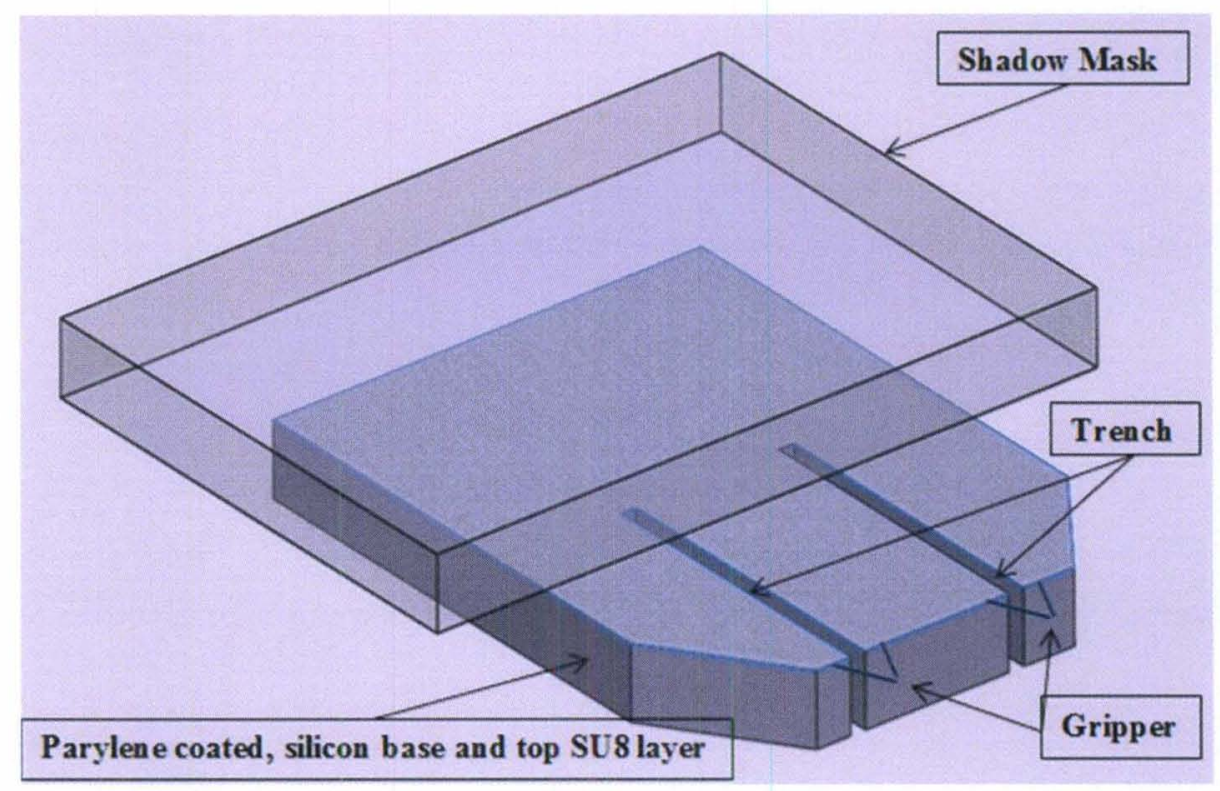

Figure 4.4 Trench - Shadow Mask Method

SU8 layer uncovered with shadow mask and the side wall of front part of trenches will be coated with chromium and silver. Since the ends of trenches are covered by shadow mask and side wall is protected with parylene, chromium and silver cannot electrically short the two arms. After sputter-coating, shadow mask is separated from the device wafer and bases with grippers are picked one by one to the collection box and stored in vacuum environment to avoid oxidation (Figure 4.5). 


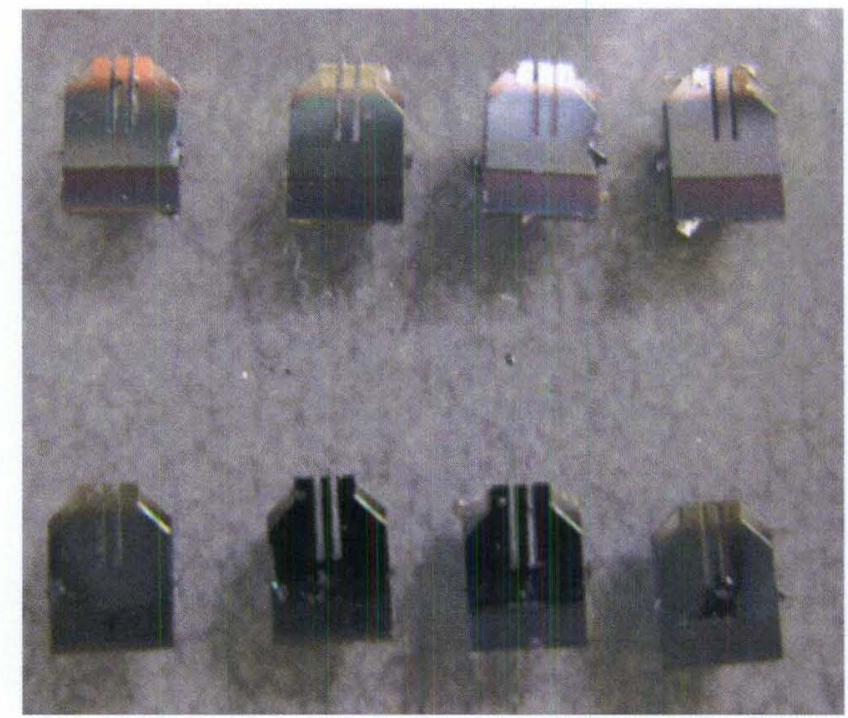

Figure 4.5 Array of micro-gripper with selective sputter-formed silver by using trench - shadow mask method

\subsection{Nano-needle growth: gallium droplet preparation}

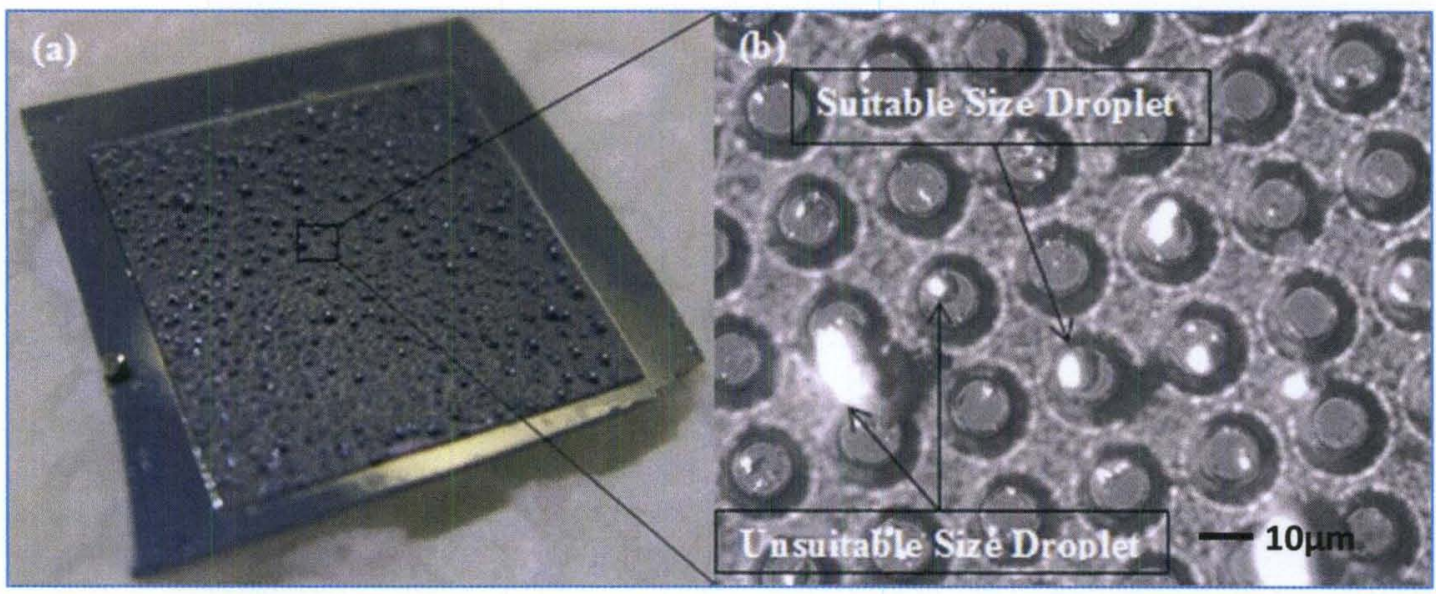

Figure 4.6 Images of: (a) Gallium droplet on top of silicon micro-pillars; (b) Close up view of suitable and unsuitable size gallium droplet. 
Prior to growing the needles, the liquid gallium is prepared into droplets to enable higher surface area to aid the needle growth. After trying several different structure substrates to hold the gallium droplet, we found that the silicon micro-pillars were quite satisfactory for holding the gallium droplets without running on the surface during needle fabrication. The spaces between micro-pillars are good place for resting an arm when growth process was carried out on the other arm. Small gallium spherical droplets are made on micro-pillars which stand on a silicon square piece. At first, a small amount of gallium is dropped on the silicon piece's surface by using a soft brush and the brush scratches on the substrate to make sure gallium is covered most area of micro-pillars' surface. Then, the substrate with gallium is dipped in $5 \% \mathrm{HCl}$ solution at $60{ }^{\circ} \mathrm{C}$ for $15-$ 30 seconds. The sample is then rinsed with deionized water and blown dry with nitrogen, also heated for $1 \mathrm{~min}$ at $60^{\circ} \mathrm{C}$ hotplate. Then by using microscope, gallium droplets are investigated to ensure good droplet generation as shown in Figure 4.6. From the microscope one can see plenty of small droplets as shown in Figure 4.6 (b), for the growth of nano-needles and these gallium droplets are usually smaller than $20 \mu \mathrm{m}$ in diameter.

\subsection{Grow $\mathrm{Ag}_{2} \mathrm{Ga}$ nano-needle on gripper arms}

Experimental set up is shown in Figure 4.7. First, micro-gripper's base is stuck to the end of the micro-manipulator arm (PPM 5000 from WPI) by using two-side tape and make sure the gripper's tips face downwards. The grippers are then aligned in horizontal plane to the location over the gallium droplets roughly using an optical microscope. 
Second, by using the horizontal digital camera (VH-Z450 from Keyence), a suitable target gallium droplet was chosen and then the tips of the grippers and that of the droplet are aligned to be along the same vertical axis to ensure contact of the gripper tips with gallium. Then the silver coated tip is inserted gently into the gallium droplet and partially retracted from the droplet forming a meniscus between the gripper and the droplet. Gallium reacts with silver film and dissolves it, and nano-needles are formed during the retraction process.

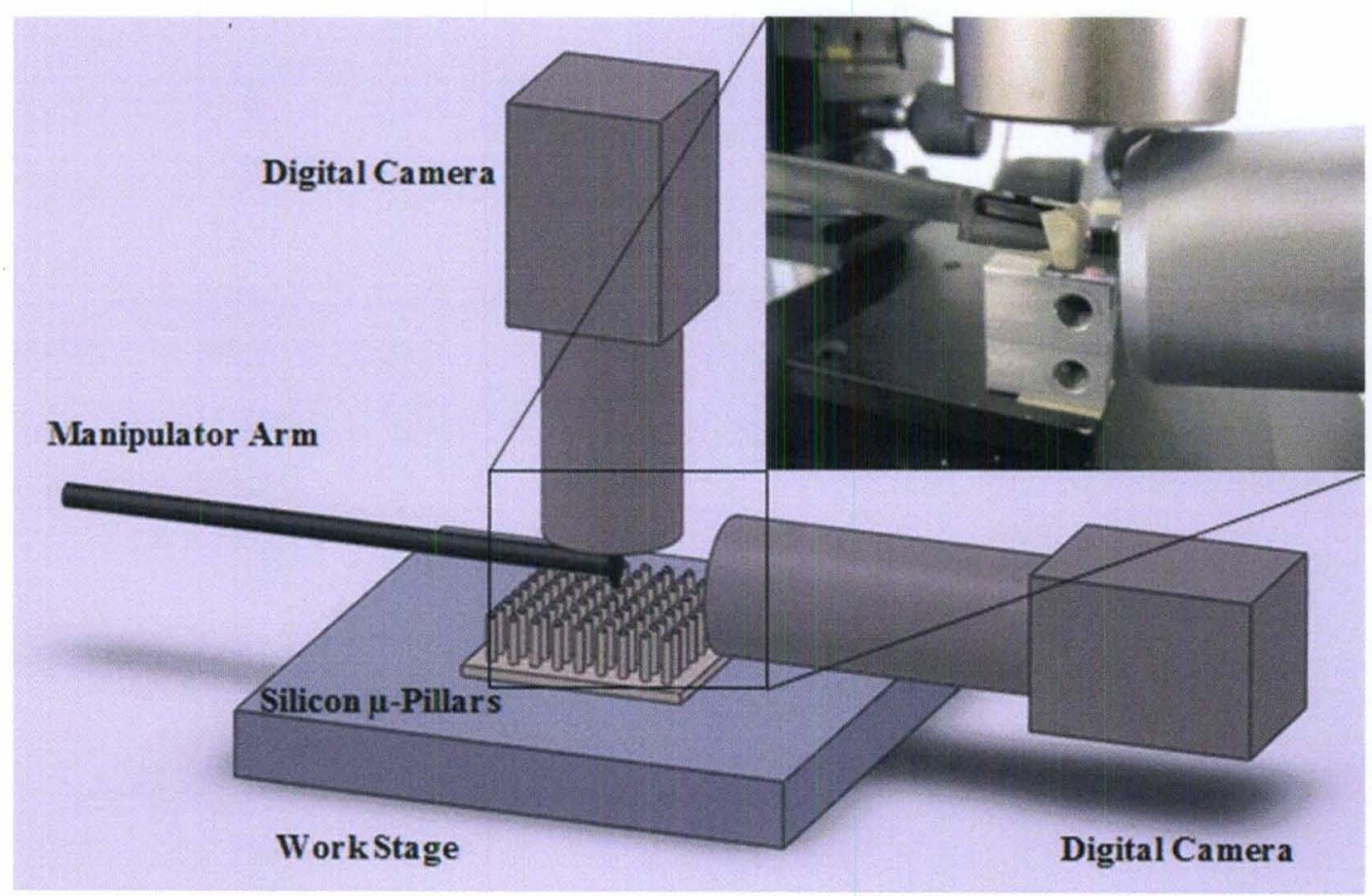

Figure 4.7 Schematic of the experimental Set-up for growth of nano-needle on the arms of the micro-grippers, inset shows a photograph of the equipment 


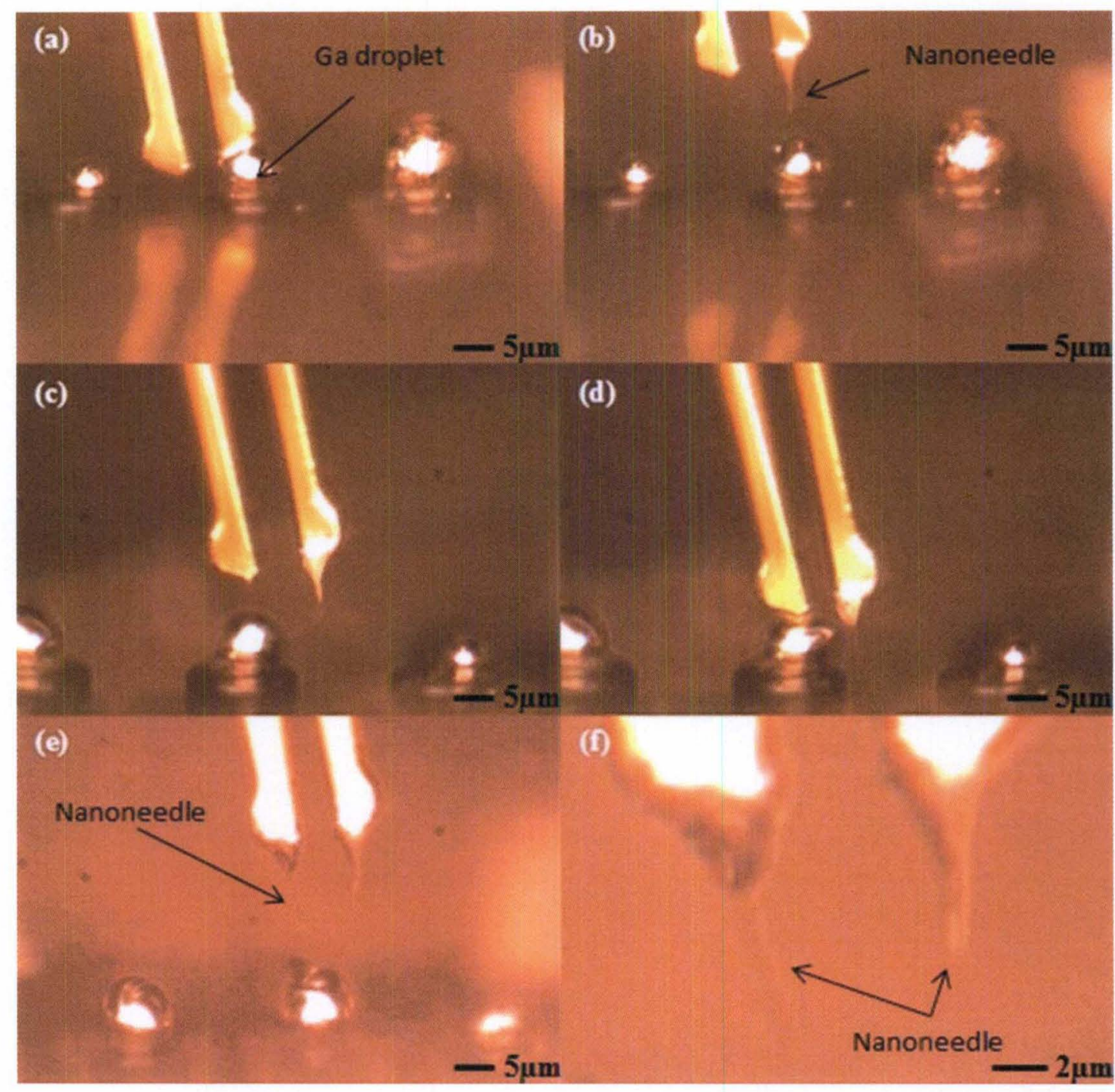

Figure 4.8 Sequential optical micrographs to grow nano-needles on gripper tips: (a) the tip of right arm is inserted into the gallium droplet, $\mathrm{Ag}$ dissolves and supersaturates the gallium; (b) retract the right tip and $\mathrm{Ag}_{2} \mathrm{Ga}$ nano-needle nucleate and grow; (c) choose another suitable gallium droplet for the left tip; (d) the tip of right arm is inserted into the gallium droplet, $\mathrm{Ag}$ dissolves and supersaturates the gallium; (e) retract the left tip and $\mathrm{Ag}_{2} \mathrm{Ga}$ nano-needle nucleate and grow; (f) closeup view of the nano-needles on the front of nano-gripper. 
Figure 4.8 presents the optical micrographs of the sequence of the needle formation process on the arms of the grippers. As one can see, the tip is pulled away a little bit from the gallium droplet to narrow the meniscus. The nano-needles continue to grow inside the meniscus and towards the center of the gallium droplet to form a single needle eventually. Finally, the tip is completely retracted and separated from the droplet and a freshly grown needle is formed attached to the tip along the pulling direction (Figure $4.8(\mathrm{a}, \mathrm{b}))$. After growth of the nano-needle on one arm, a second fresh gallium droplet is chosen to grow nano-needle on the other arm (Figure 4.8 (c, d and e)). The total time of needle formation on the gripper's tip ranges from 2 minutes to 4 minutes.

\subsection{Wire-bonding}

Following successful growth of nano-needles, we used a piece of side-brazed dual in-line ceramic package (SPECTRUM Semiconductor Materials, INC.), with an open side and 3 pins for packaging and wire bonding the nano-needles. Again wire bonding here presents challenges as the traditional techniques such as ball bonding cannot be applied here due to the high temperatures involved. Sameoto et al., present a method to bond $100 \mu \mathrm{m}$ SU8 film MEMS structure by using ball bonding [57]. For our case, since

the gripper material is a thin layer of SU8, $8 \mu \mathrm{m}$, which cannot stand longer in high temperature around $110^{\circ} \mathrm{C}$ like $100 \mu \mathrm{m}$ thick SU8 film, avoiding plastic deformation, traditional ball bonding [58] cannot be applied here. Flip Chip bonding was applied to SU8 chip bonding by A Johansson et al., however it is lack of manual installation, needs 
flat surfaces to mount and the short connections are quire rigid for gripper applications [59]. Finally, silver as a layer is not compatible with wedge bonding techniques.

The silicon chip coated with paralyene is bonded to the package by using nonconductive cyanoacrylate. Three $0.5 \mathrm{~cm}$ long and $25 \mu \mathrm{m}$ diameter straight gold wires are cut out from pre-fabricated wire rolls. The wires were then bonded to the silver layer using silver epoxy (\#H20E, Epoxy Technology Inc.), a kind of conductive glue, is transferred to junction area for bonding under optical microscope. After bonding, the device is cured inside oven at $60^{\circ} \mathrm{C}$ for 1 hour, and then is left in ambient temperature for $12 \mathrm{hrs}$. The optical micrograph of the entire packaged device is presented in Figure 4.9.

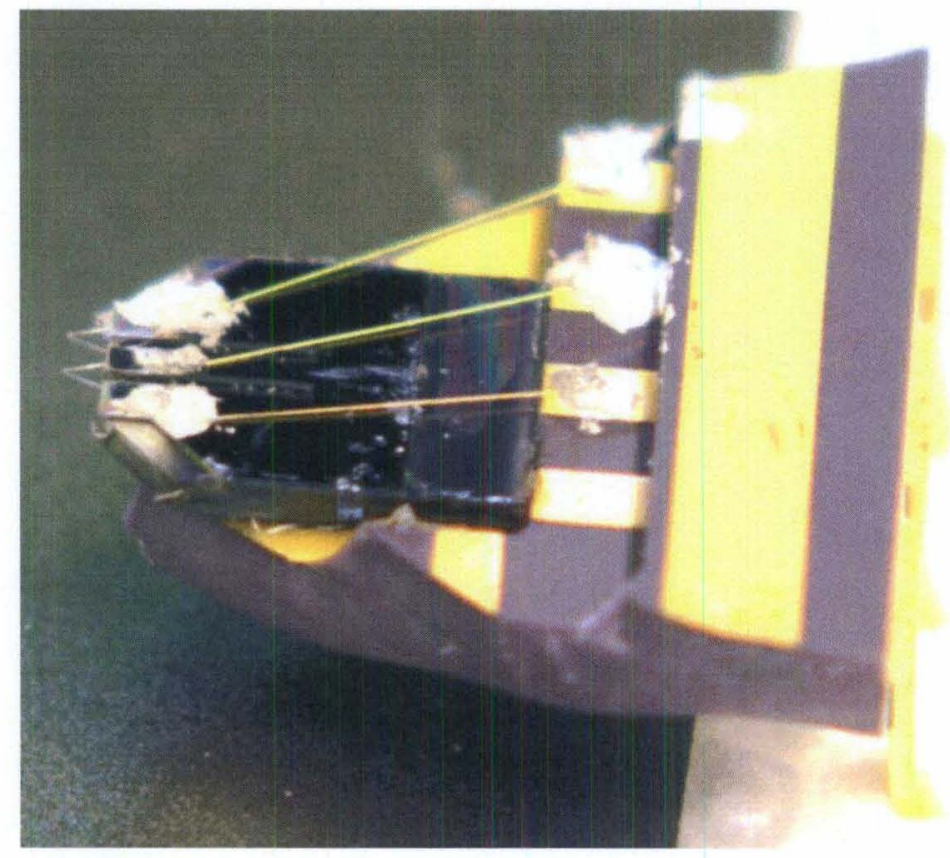

Figure 4.9 Image of the package after wire-bonding 


\section{CHAPTER 5 - CHARACTERIZATION OF NANO-NEEDLES AND MEASUREMENT OF MATERIAL PROPERTIES}

5.1 Characterization of devices

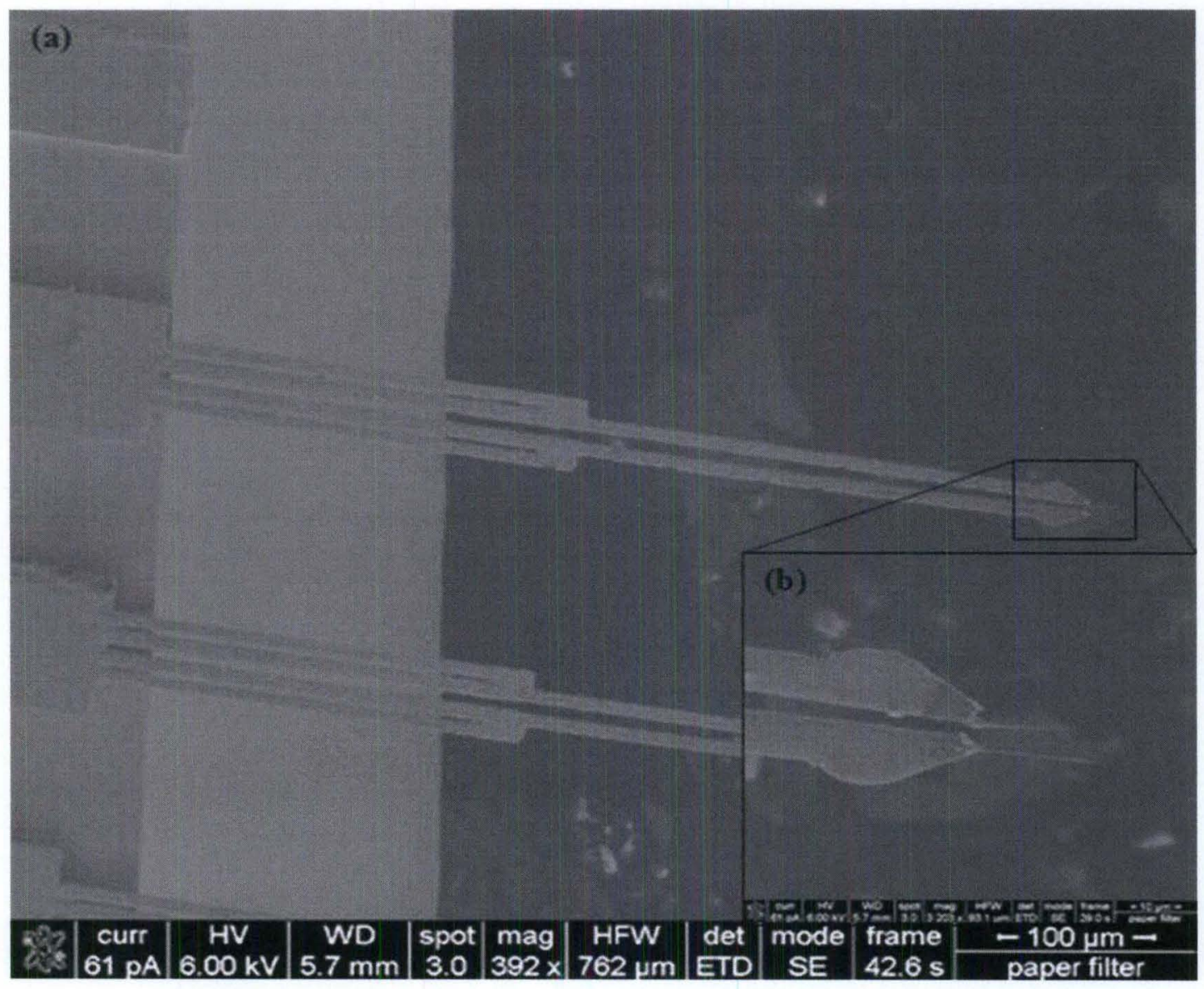

Figure 5.1 SEM images of the first design of nano-grippers: (a) full view; (b) close up view. 


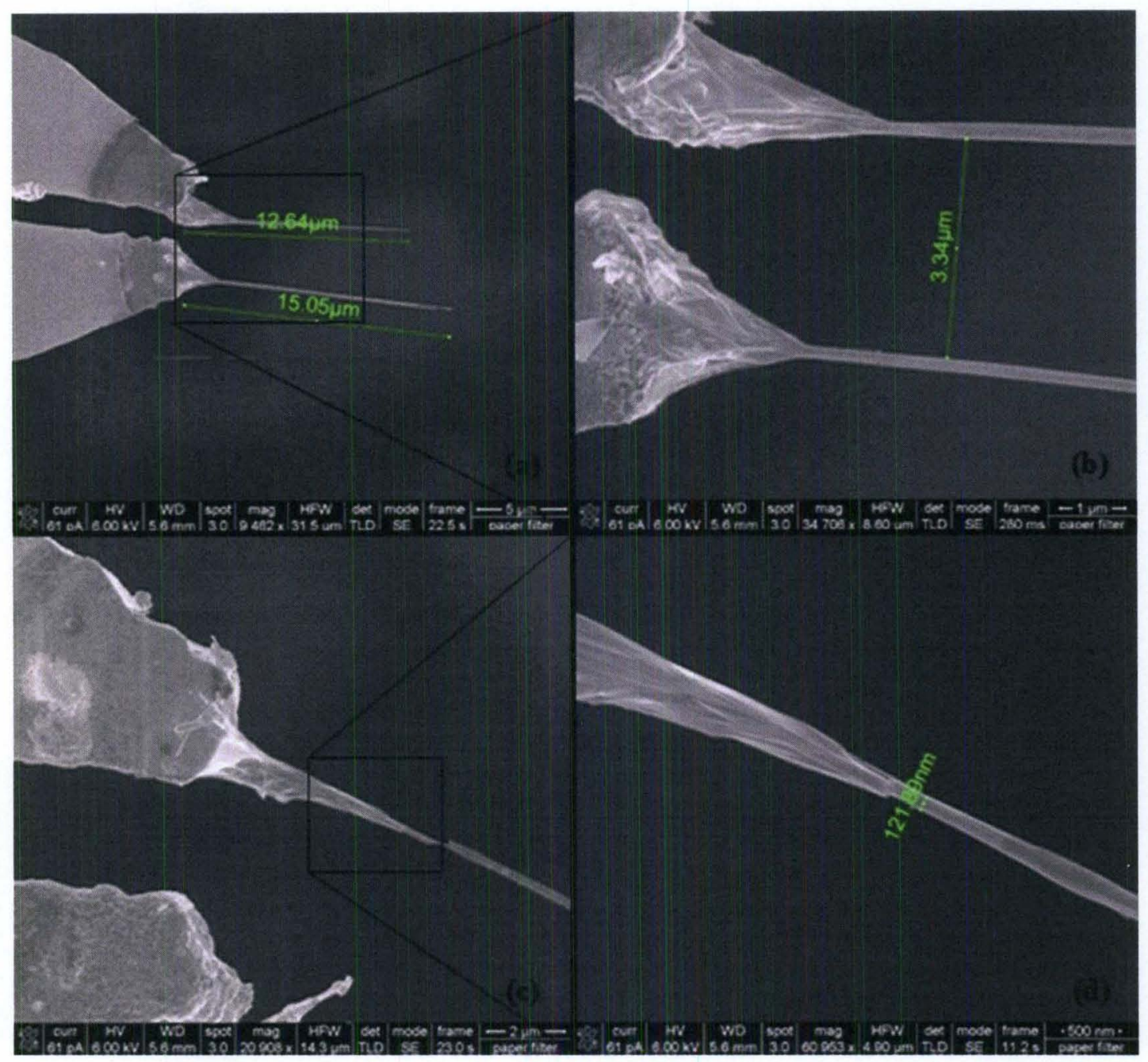

Figure 5.2 SEM images of nano-needles grown on tips of micro-gripper

Nano-needles grown on the grippers are first characterized using a scanning electron microscope for measurement of length and diameter of the needles. Figure 5.1 presents the pair of nano-needle electrodes from the first design. The inset in the Figure 5.1 presents the actual needles coming out of the arms of the gripper. SEM characterization of these needles is shown in Figure 5.2 measured $12-15 \mu \mathrm{m}$ long and 120 $\mathrm{nm}$ in diameter is integrated with micro-gripper. The distance between the needles between adjacent arms was measured to be $\sim 3.3 \mu \mathrm{m}$. 
Figure 5.3 presents the nano-needles coming out of the arms of second gripper design. Figure 5.4 presents the SEM image of a nano-needle shown in Figure 5.3. The inset is the micrograph presents the needle diameter of $\sim 190 \mathrm{~nm}$.

These SEM image shows that the nano-needles can be grown approximately to the similar length and diameters in different gripper designs.

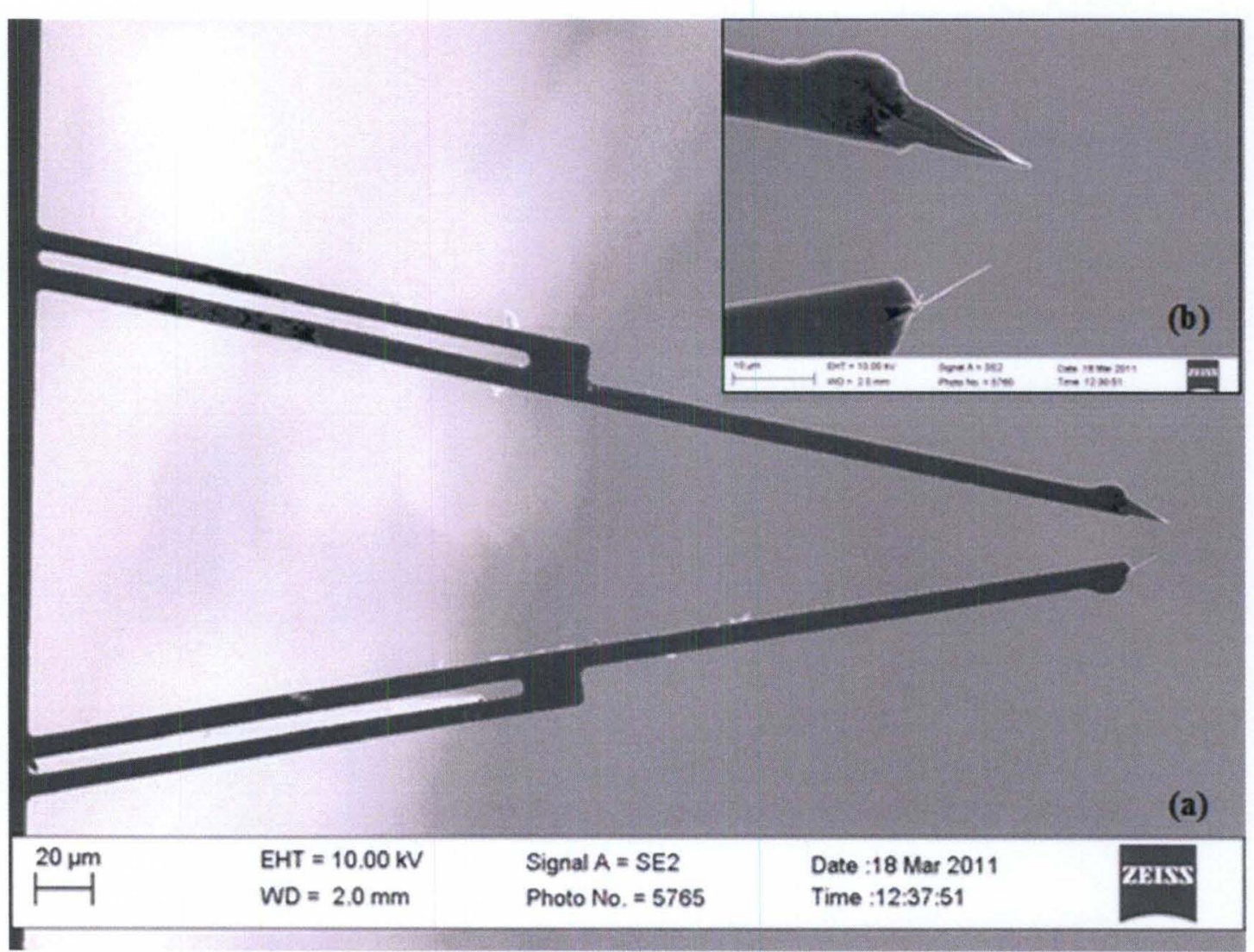

Figure 5.3 SEM image of the second design of nano-grippers: (a) full view; (b) close up view. 


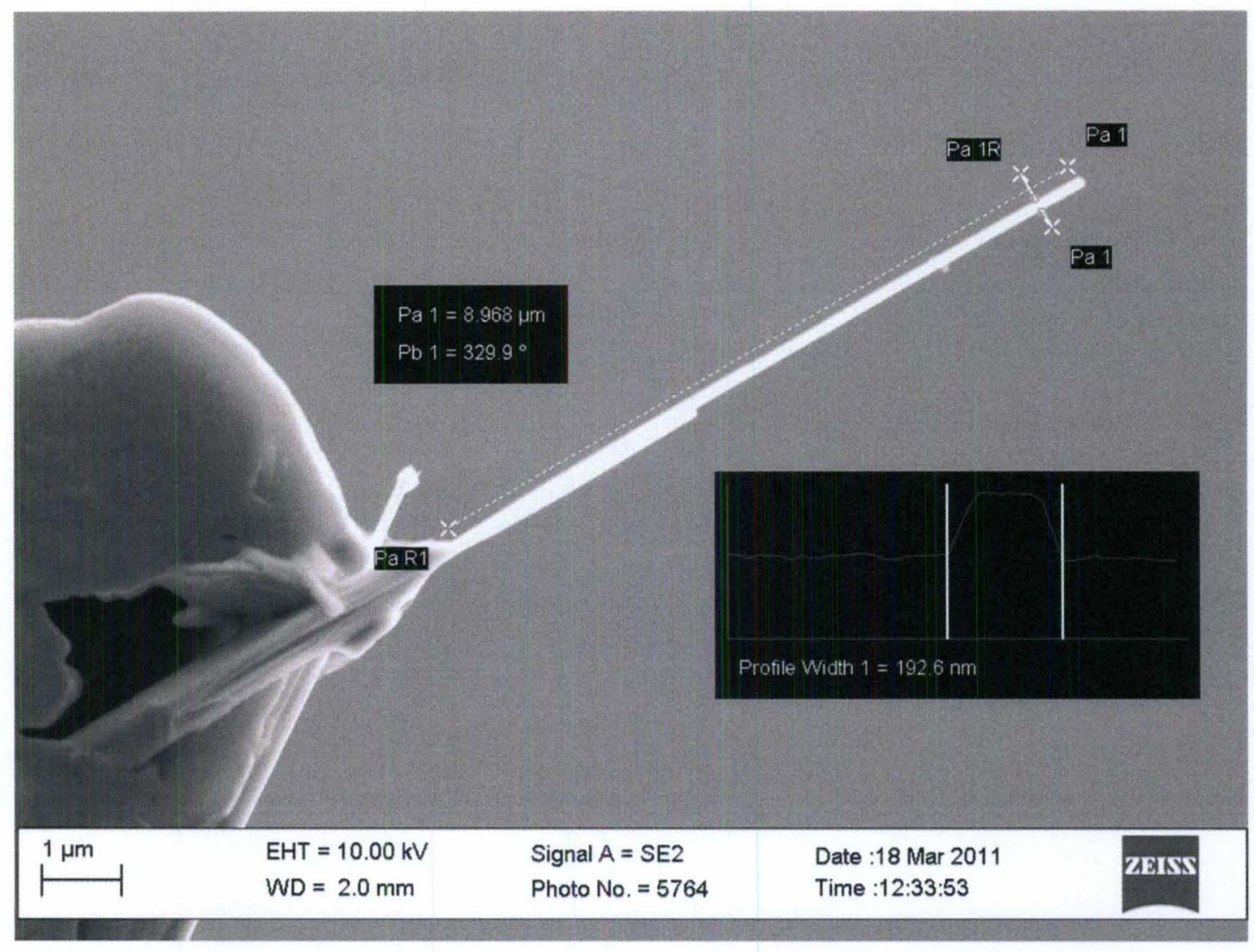

Figure 5.4 SEM image of a nano-needle integrated with a micro-gripper tip

\subsection{Measuring electrical property of carbon nanotube film}

In order to demonstrate the capability of the nano-needle, we first tried to measure the electrical property of a small carbon nanotube film that was prepared by vacuum filtration process. The packaged nano-needles were connected to precision semiconductor parameter analyzer (F4156C, Agilent Technologies) to see the efficacy of measurement of electrical properties of nano-materials. As a demonstration, the surface electrical properties of a small carbon nanotube film were investigated. 


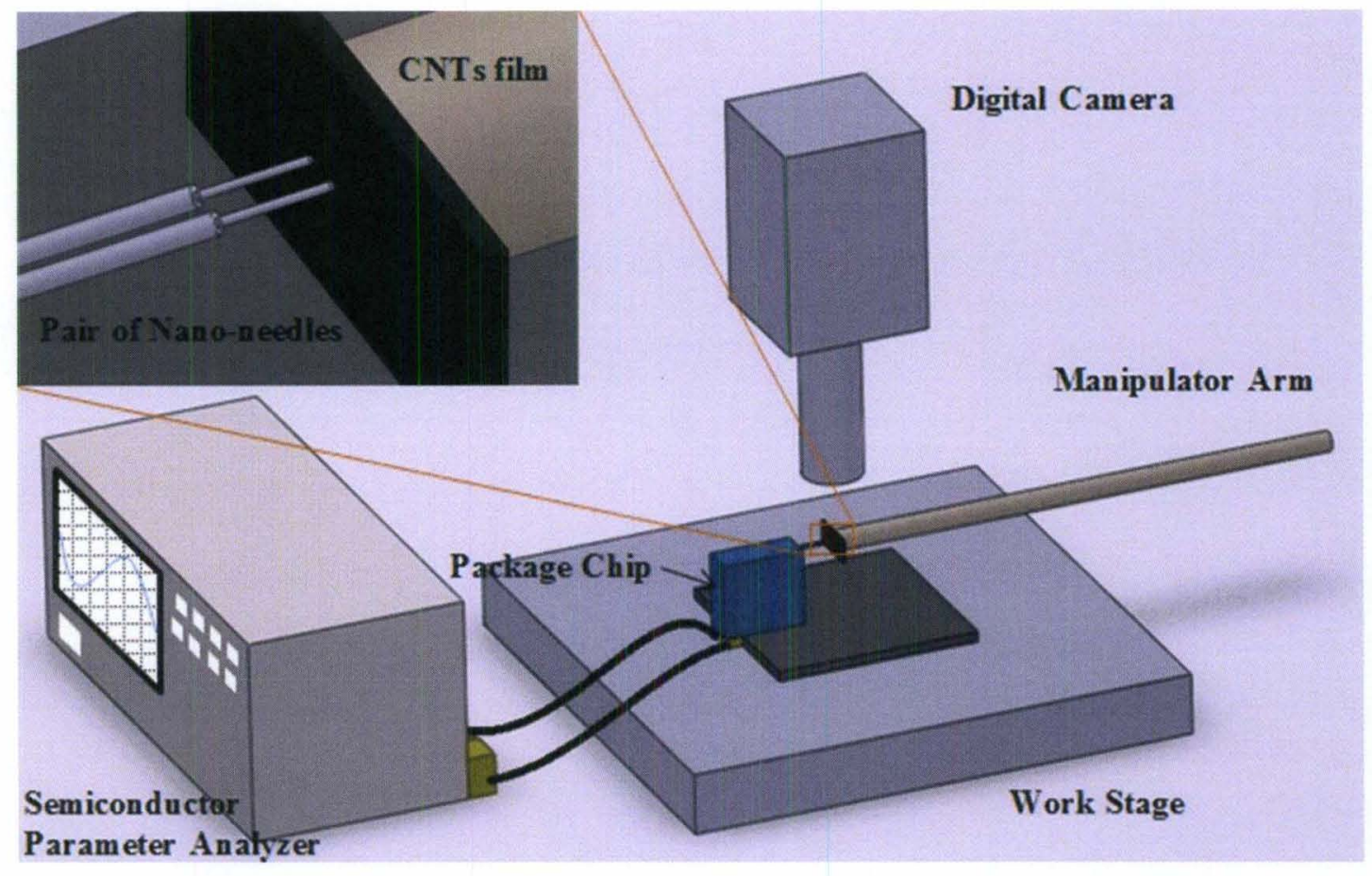

Figure 5.5 Schematic of the experimental set-up for measuring the CNTs film's surface

Figure 5.5 presents the experimental set-up for demonstration of the electrical property measurement on carbon nanotube film. First, the chip that the nano-needle electrodes on the grippers were packaged on is connected to the semiconductor parameter analyzer and anchored to the work stage. Second, a $10 \mu \mathrm{m}$ thick free standing single wall CNTs film made by vacuum filtration (HiPCO, NanoIntegris Inc.) is bonded to the probe tip that is connected to micro-manipulator. The probe tip is then slowly brought close to the needles using the X-Y-Z micro-manipulator using an optical microscope. Current versus time was measured continuously at constant voltage of $0.2 \mathrm{~V}$ during the start of the experiment. Time steps and total period are set $0.2 \mathrm{~s}$ and $120 \mathrm{~s}$ respectively. During the entire experiment, the probe tips with the carbon nanotubes were brought in contact 
for 10-20 s and followed by retraction. The entire experimental data was recorded using Lab View software. We did this several times to measure that the current versus time from the nanotube film and to see the jumps in current during contact. Our assumption was that an intimate contact between a sharp metallurgical junction such as the nanoneedle and the nanotube film would result in sudden jump in the current measured as a function of time. During the first sweep period, the pair of nano-needle electrodes does not touch the CNT film. Figure 5.6 presents the current versus time for the nano-needle. The value of current recorded was $\sim 10^{-12}$ amps showing no contact. Some spikes are seen in the graph that could be coming from local surface disturbance or vibration of the needle. However, the overall pattern is that when the needles do not touch each other the current values are similar to open circuit.

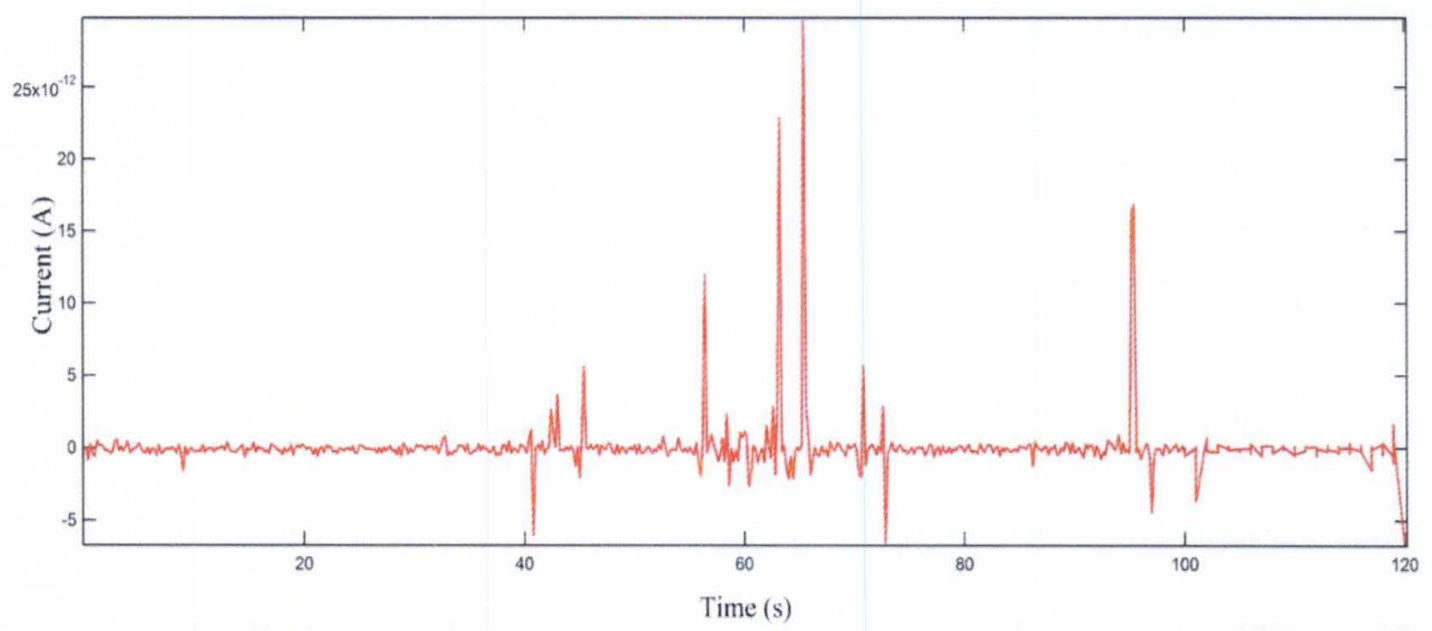

Figure 5.6 Current - Time curve for non-contact mode between nano-needle electrode and CNT film 
Figure 5.7 presents the current versus time for three consecutive contact and noncontact mode of operation. As per our assumption, one can see large jumps in the current when brought into contact. These currents are also repeatable over three cycles. The solid line represents the curve fitting of the actual data. The data markers present the actual data. One can see that in contact mode, the current stabilizes for 10-20 seconds which was approximately the time of contact.

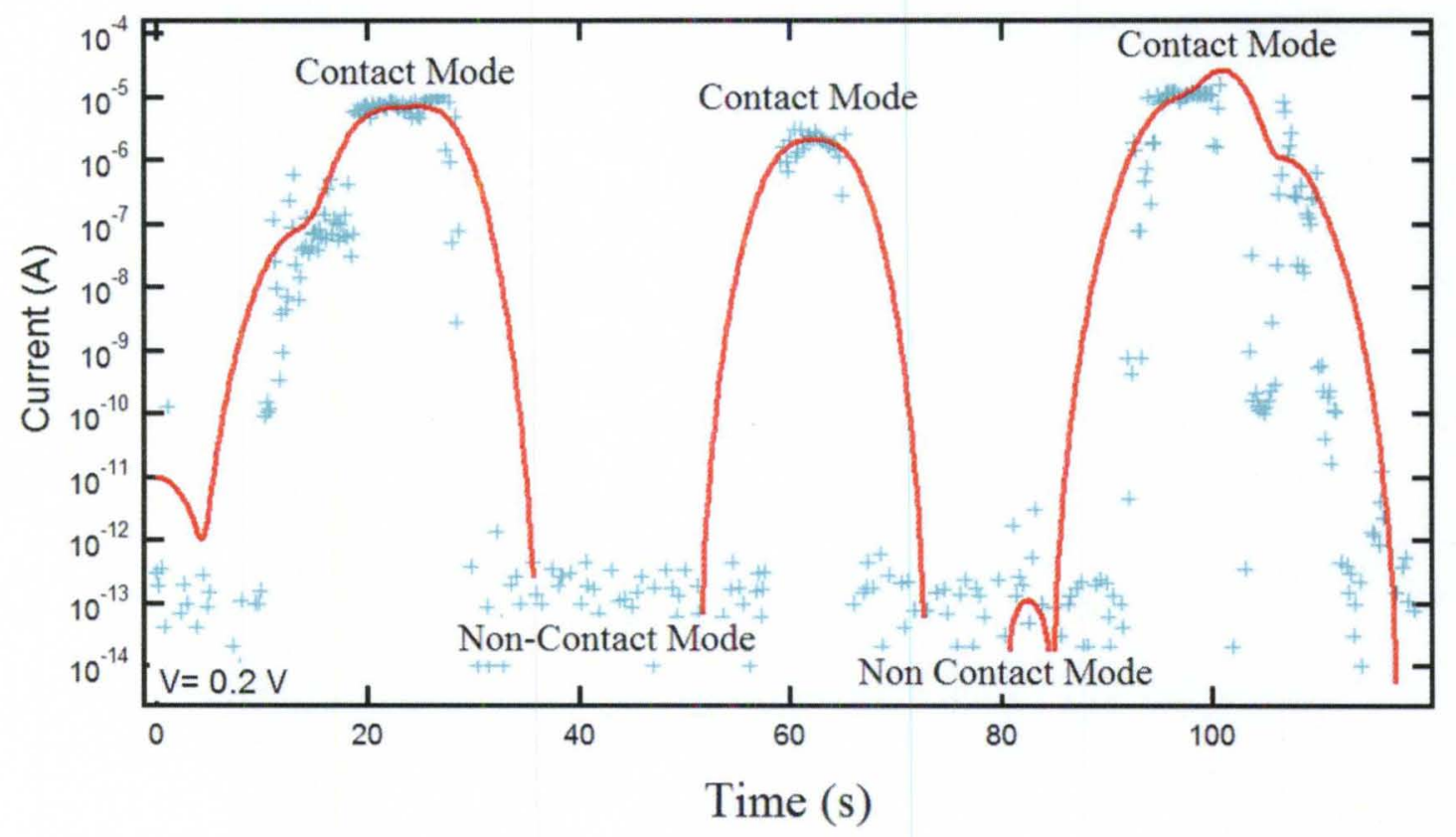

Figure 5.7 Current - Time curve show the huge change between contact mode and non-contact mode of the pair of nano-needle electrodes and the surface of CNTs film.

When the tip was retracted, the current jumped down very rapidly showing that the nano-needles were not touching each other as in Figure 5.7. The current in figure 5.8 during contact mode jumped from $10^{-11}$ amps to $10^{-5}$ amps demonstrating 6 orders of magnitude jump in current. For a constant voltage of $0.2 \mathrm{~V}, \mathrm{R}_{\mathrm{on}}=20$ Kilo-ohms to $\mathrm{R}_{\text {off }}=$ 
2 Tera-ohms. They demonstrate the huge on/off ratio of the nano-needle electrodes for material property measurement. The $\mathrm{R}_{\text {on }}$ measurement of 20 Kilo-ohms also gives us great confidence in our measurements as carbon nanotube films of couple of micron thickness has resistances in the area of 1-100 kilo-ohms depending on their thickness [6063]. The average ratio between non-contact resistance and contact resistance reaches $5.7 \times 10^{7}$ (Table 5.1 ). Such extraordinary change in resistance shows the high sensitivity of the nano-needles that is suitable for monitoring small molecules.

Table 5.1 Average resistance ratio between non-contact mode and contact mode of the pair of nano-needle electrodes and CNTs film's surface

\begin{tabular}{|c|c|c|}
\hline No. & $\begin{array}{c}\text { Ratio } \\
\left(\mathrm{R}_{\text {NON-CONTACT }} / \mathrm{R}_{\text {CONTACT }}\right)\end{array}$ & Ratio AVERAGE \\
\hline 1 & $5 \times 10^{7}$ & $5.7 \times 10^{7}$ \\
\hline 2 & $2 \times 10^{7}$ & \\
\hline 3 & $1 \times 10^{8}$ & \\
\hline
\end{tabular}

One cycle of current change between non-contact mode and contact mode is show in Figure 5.8. It should be noted that these measurements were made using a manual manipulator. Automatic manipulators where the probe tip with the nanotube film driven by motorized stages can result in precise change in current at the pre-determined time. Nevertheless, our measurements show the versatility of using the nano-needles for material property measurement. Not only the experiment shows high sensitivity of the device, but also shows the device's repeatable ability. Since the pair of nano-needle 
electrodes is integrated with optically actuated micro-gripper, in the future the electrical property measurement of single wall carbon nanotube and nano-wires becomes a possibility.

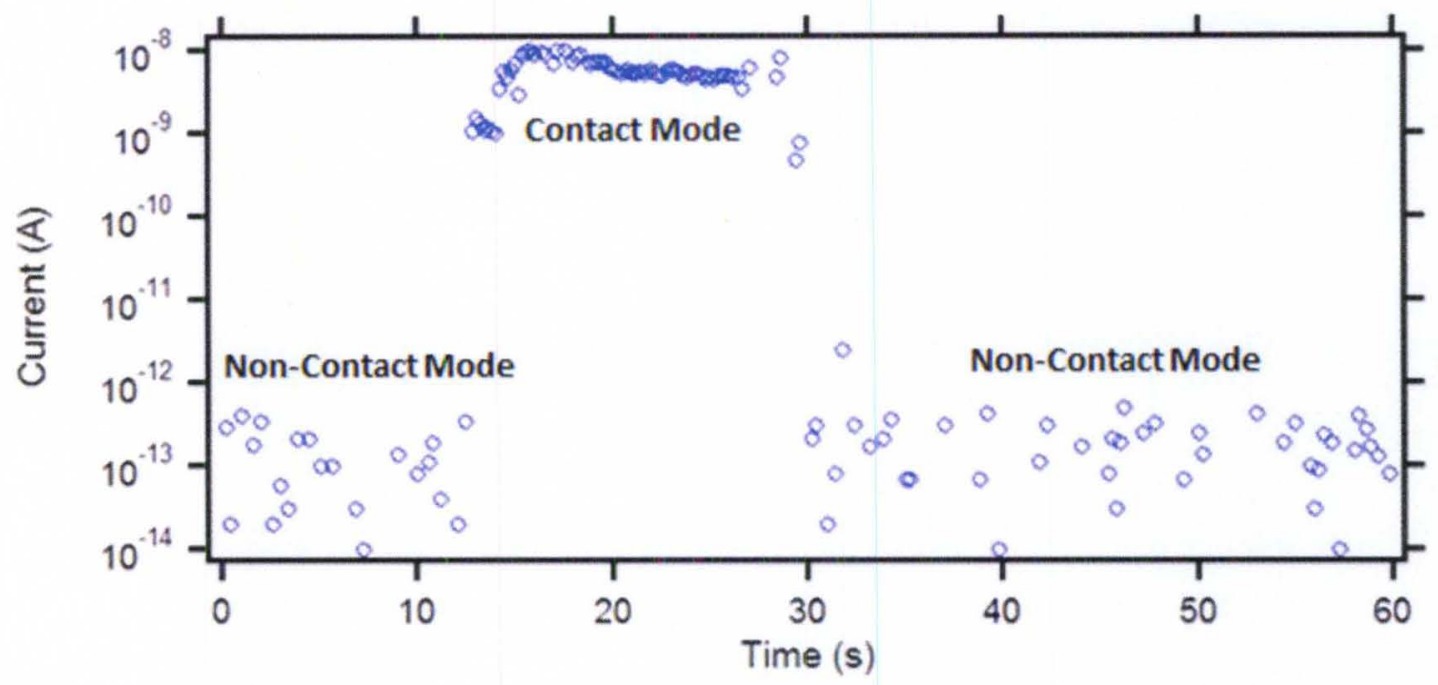

Figure 5.8 One cycle of current change between non-contact mode and contact mode between the pair of nano-needle electrodes and CNT film surface. There is five orders of jump in the current $\left(10^{-13}\right.$ to $\left.10^{-8} \mathrm{~A}\right)$

\subsection{Electrical property measurements in liquids}

In order to estimate the electrical property measurements of the device in wide variety of environments, we measured the I-V characteristics of phosphate buffered saline (10X, PH7.4, Mediatech Inc.). The experimental set-up was the same as measuring the electrical properties of CNTs films. The pipette was tipped on the probe tips with a drop 
of PBS at the tip of the micro-pipette supplying about $0.04 \mathrm{ml}$ phosphate-buffered saline at the end of manipulator (Figure 5.9).

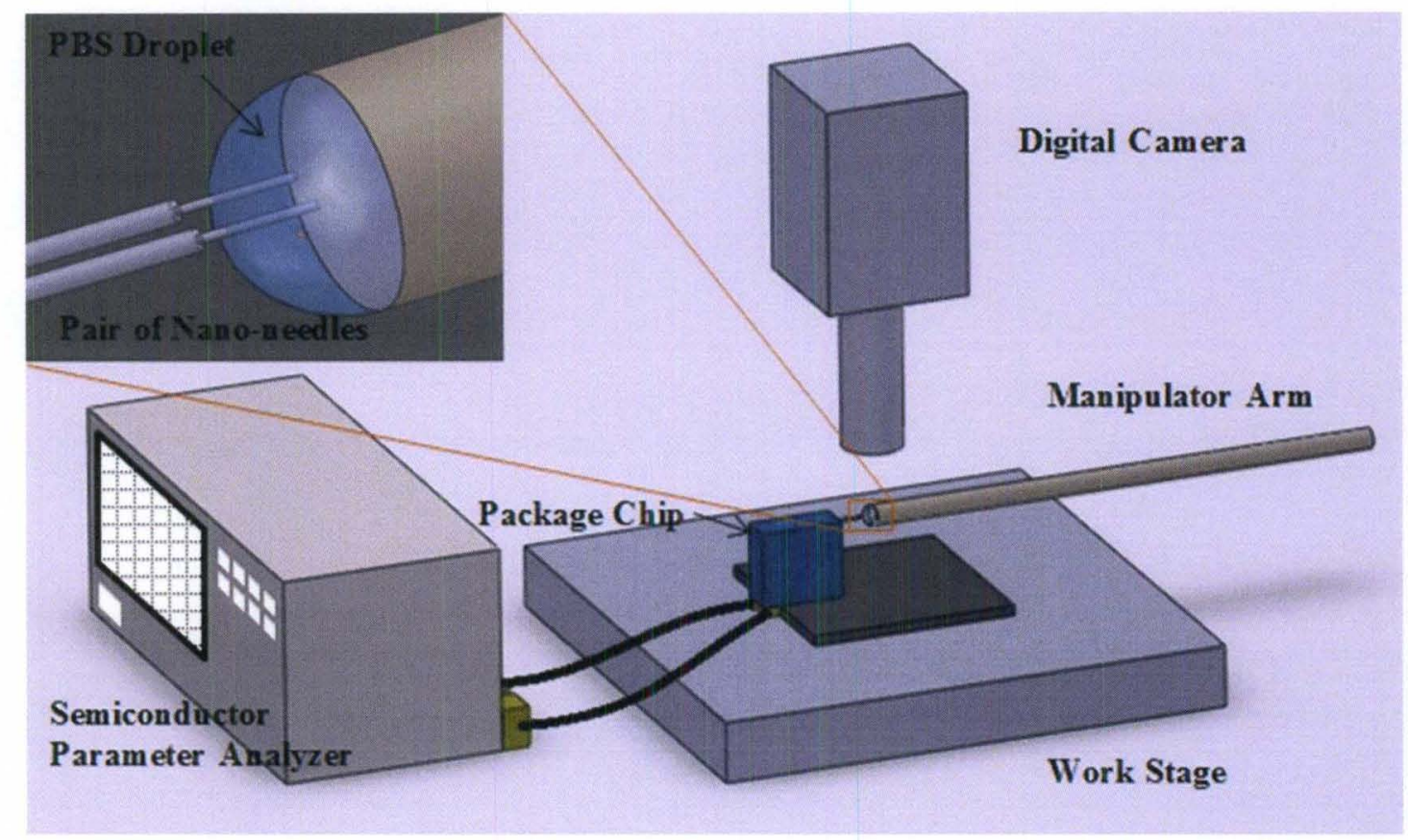

Figure 5.9 Schematic of the experimental set-up for measuring the electrical properties of PBS

First, I-V curve of non-contact mode measured by precision semiconductor parameter analyzer shows open circuit resistance is around 120 Gig-Ohms from Figure 5.10 which shows that the gripper tips are insulated for electrical properties' measurement in ionic liquids. Following the electrical measurement in open circuit condition, the manipulator arm was tuned in manually to make the PBS droplet touch the pair of nano-needle electrodes. Again there was 5-6 orders of magnitude jump in current from non-contact mode to when the liquid contact occurs as shown in Figure 5.10. 
Electro-chemical reactions between PBS droplet and nano-needle could potentially result in unique changes in current. In order to investigate surface reactions at the electrodes, we conducted linear sweep voltammetry and monitored current-voltage for oxidation and reduction of the electrode surface. 


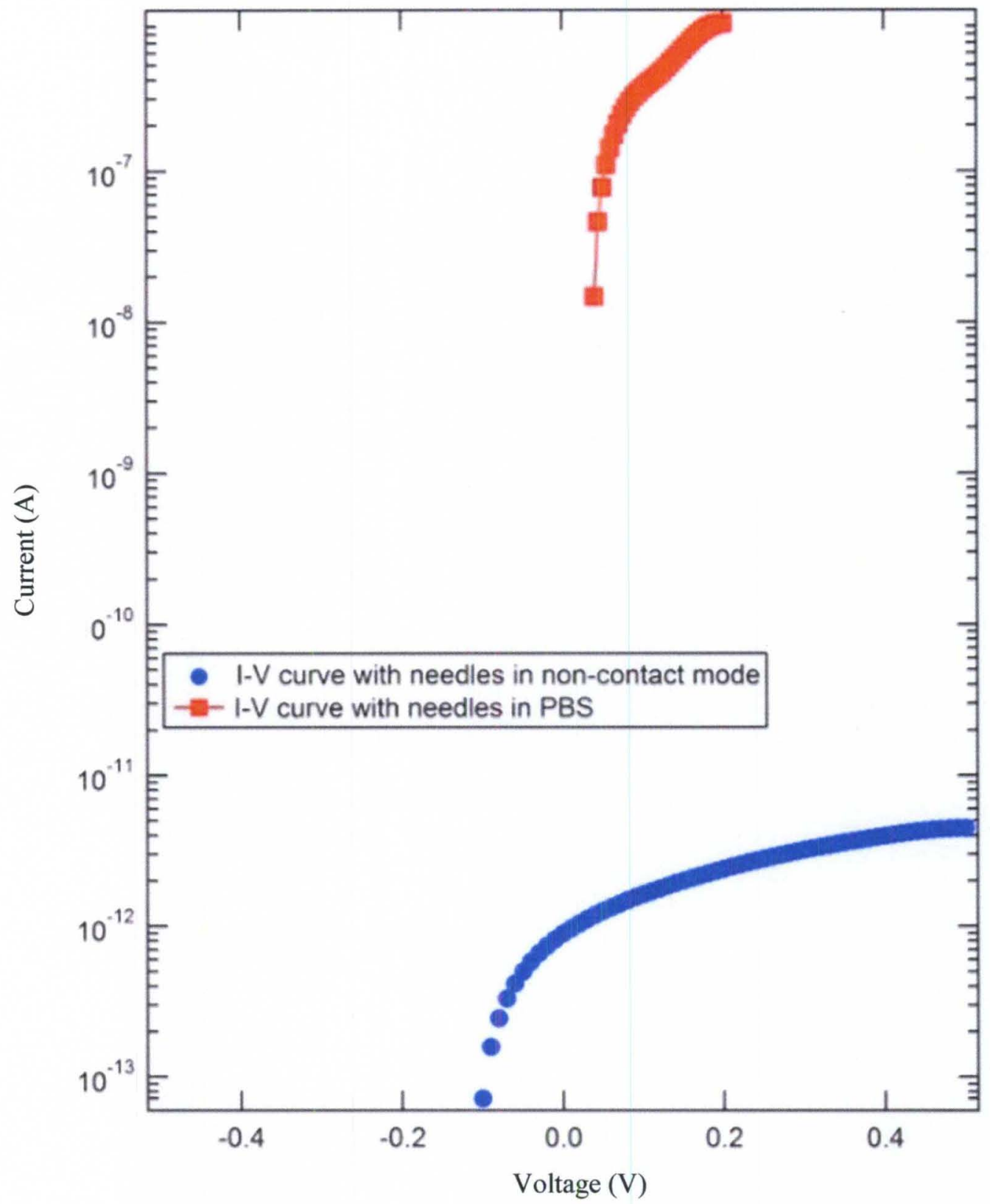

Figure 5.10 I-V curves when two electrodes in non-contact mode and inside of PBS

In order to clear this problem, we performed a forward and backward Linear Sweep Voltammetry (LSV) measuring the current-voltage between the nano-needles 
similar to cyclic voltammetry. Ten sweeps use ten different scanning rates between $(40 \mathrm{mV} / \mathrm{s}-12 \mathrm{mV} / \mathrm{s}$, Figure 5.11) from $-0.2 \mathrm{~V}$ to $0.2 \mathrm{~V}$.

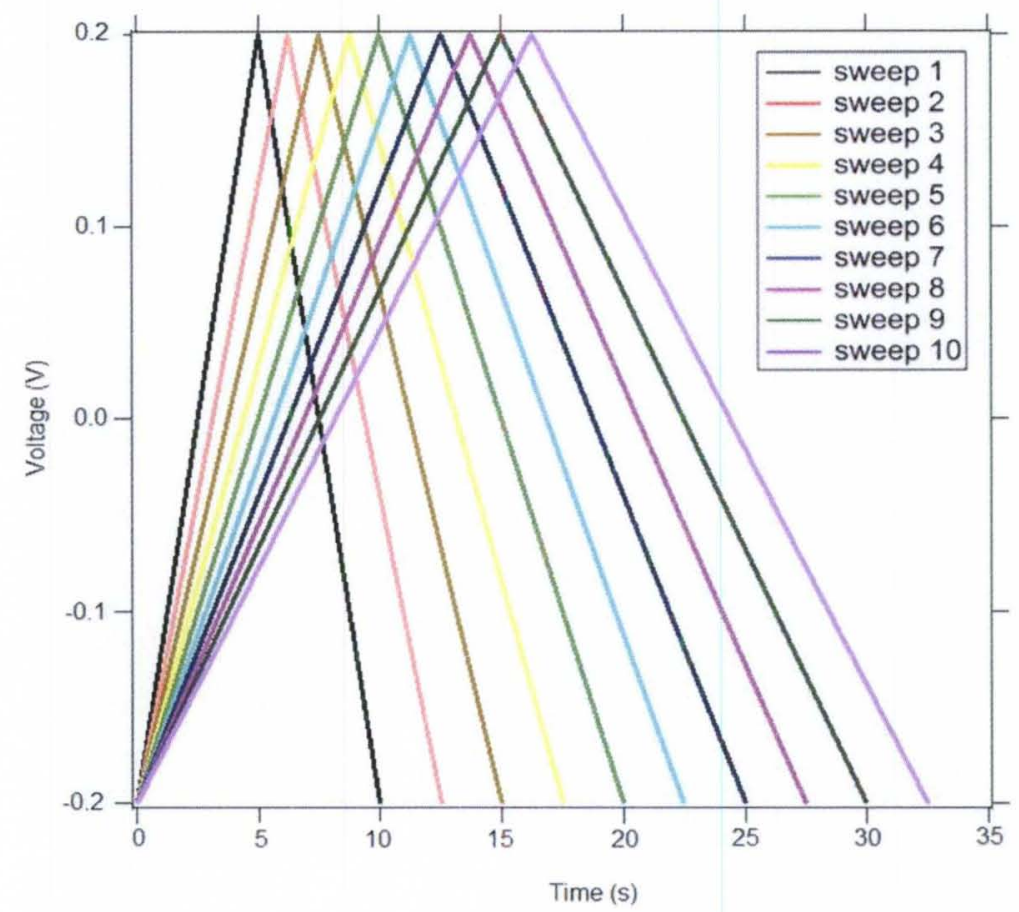

Figure 5.11 Voltage linear change vs. time for ten sweeps' measurement

Forward and backward LSV curves with different voltage scanning rate are shown in Figure 5.12. From the curve, we can see a sharp peak at $\mathrm{E}=0.11 \mathrm{~V}$, when voltage linearly change from $-0.2 \mathrm{~V}$ to $0.2 \mathrm{~V}$, which is the reductive peak. When voltage linearly change from $0.2 \mathrm{~V}$ to $-0.2 \mathrm{~V}$, we see a peak at $\mathrm{E}=-0.11 \mathrm{~V}$, which is the oxidation peak. Every sweep with certain voltage scanning rate, the ratio between reductive peak current ipc and oxidation peak current ipa has a certain value $\sim 1$. This illustrates that the electrochemical reaction between electrode and PBS is reversible or quasi-reversible [64]. We also can find, with different voltage scanning ratio, the peak currents occur at the 
same voltage, which means the reactions of pair nano-needle electrodes have rapid electron transfer kinetics.

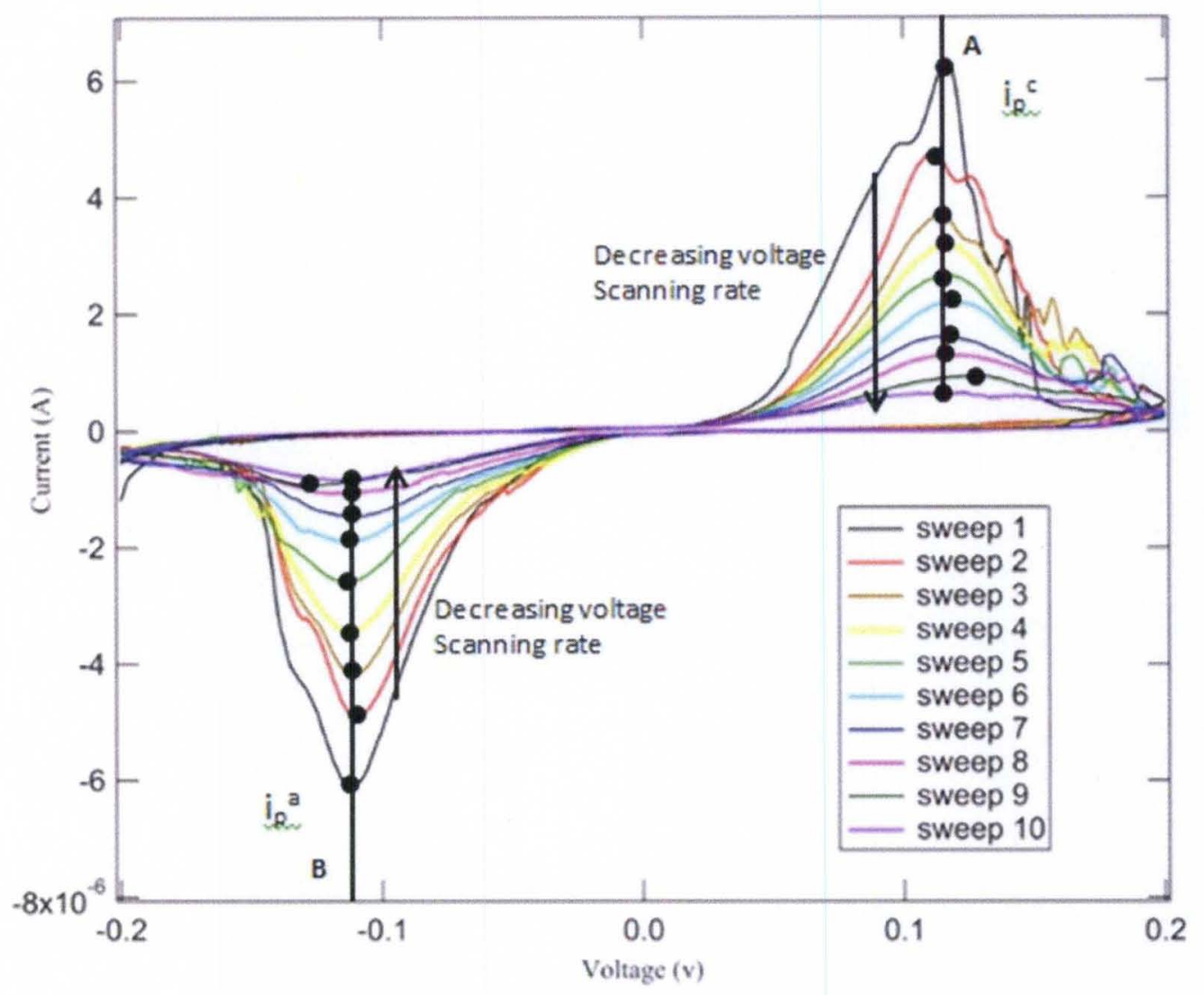

Figure 5.12 Linear Sweep Voltammetry of PBS with different voltage scanning rates (Scan rates of sweep curves (1-10) correspond to $40-12 \mathrm{mV} / \mathrm{s}$ )

The scan process begins from the left hand side of the current/voltage plot where no current flows. As the voltage is swept further to the right (to more reductive values) a current begins to flow and eventually reaches a peak before dropping. To rationalize this behavior we need to consider the influence of voltage on the equilibrium established at the electrode surface. Here the rate of electron transfer is fast in comparison to the 
voltage sweep rate. Therefore at the electrode surface equilibrium is established identical to that predicted by thermodynamics. The exact form of the curve can be rationalized by considering the voltage and mass transport effects. As the voltage is initially swept from the initial value the equilibrium at the surface begins to alter and the current begins to flow. The current rises as the voltage is swept further from its initial value as the equilibrium position is shifted further to the right hand side, thus converting more reactant and enabling rapid mass transport. The peak occurs, since at some point the diffusion layer has grown sufficiently above the electrode so that the flux of reactant to the electrode is not fast enough to satisfy that required by the Nernst equation [65]. In this situation the current begins to drop just as it did in the potential step measurements. In fact the drop in current follows the same behavior as that predicted by the Cottrell equation [66].

Since $\Delta \mathrm{E}$ between reductive voltage and oxidation voltage from figure 5.12 is $\sim 0.22 \mathrm{~V}$.

$\mathrm{AgCl}(\mathrm{s})+\mathrm{e}-\leftrightarrow \mathrm{Ag}(\mathrm{s})+\mathrm{Cl}^{-}$(sat'd), with a value for $\mathrm{E}$ of $+0.22 \mathrm{~V}$

We suppose the $\mathrm{Ag}$ inside of $\mathrm{Ag}_{2} \mathrm{Ga}$ intermetallic has a reaction with chloride ions in PBS as the reaction function above. In order to prove our assumption, we characterize the needles by SEM shown in Figure 5.13. It seems that some other materials are deposited on the surface of $\mathrm{Ag}_{2} \mathrm{Ga}$ nano-needle. Energy Dispersive Spectroscopy (EDS) will be used later to determine the exact element of these deposit materials.

Since the $\mathrm{Ag}_{2} \mathrm{Ga}$ nano-needle will react with some ions in liquid, this type nanoneedle cannot contact with liquid directly. A solution is indicated here. First, a 100nm layer paralyene (an insulator layer) is coated on the whole surface of package besides 
gripper and nano-needles. Secondly, dip the tips of nano-needles into $\mathrm{K}_{2} \mathrm{PtCl}_{4}$ solution to exposure the end part. It seems that the $\mathrm{K}_{2} \mathrm{PtCl}_{4}$ solution diffuses through the parylene film and interacts with the $\mathrm{Ag}_{2} \mathrm{Ga}$ nano-needle. Platinum atoms exchanged with Silver and Gallium atoms and make a coating at the tip of the needle and expose the tip.

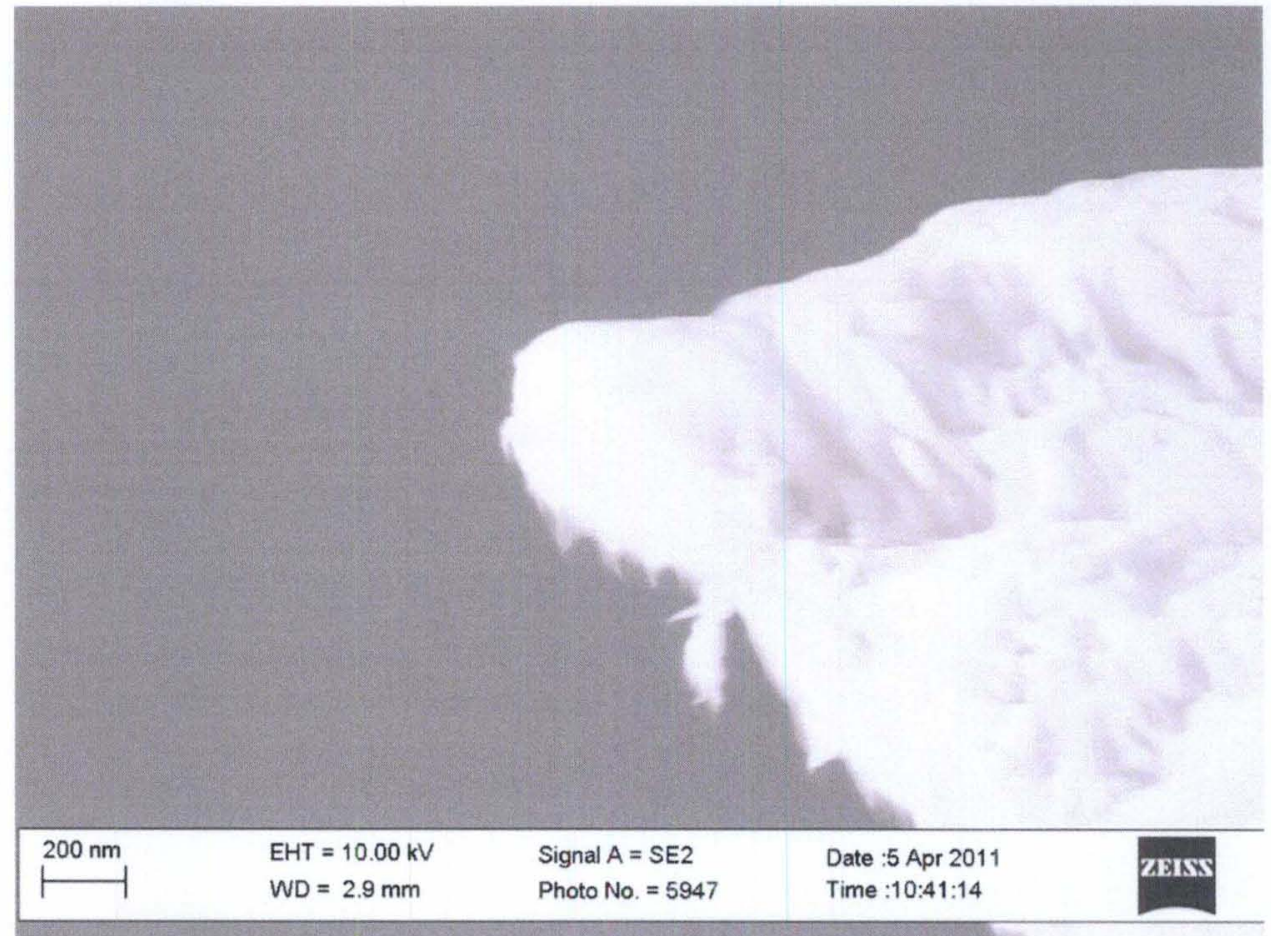

Figure 5.13 A SEM picture of nanoneedle after measurement in PBS 


\section{CHAPTER 6 - CONCLUSION AND FUTURE WORK}

In conclusion, in this thesis we explored the integration of $\mathrm{Ag}_{2} \mathrm{Ga}$ nano-needles on batch fabricated micro-grippers. The micro-grippers serve as excellent platforms for the growth of the nano-needles. Nano-needles about 12-15 microns in length and 120-190 $\mathrm{nm}$ in diameter were grown on the grippers. Wire-bonding with chip package free the device in order to move the device into $\mathrm{X}-\mathrm{Y}-\mathrm{Z}$ dimensions with real time electrical measurement of materials in air and liquids. As an initial demonstration we measured the current versus time of a small piece of carbon nanotube film and liquid PBS. I-V curve demonstrated that the device had a high resistance in non-contact mode and large resistance change when the nano-needle electrodes touch the target materials (the surface of simple CNT film and PBS). LSV curves show some electrochemical reaction is happened inside the PBS, possible between the nano-needle and Chloride ions, a solution is indicated here to solve this problem. All these demonstrations show that nano-needles are ideal platforms for measurement inside cells.

In the future, this type nano-gripper will be used to grab nano structure and particle. Different cell electrochemical measurements not only in air but also in liquid will be applied by the device. This device can be used to measure electrical properties of 
nanowire and single carbon nanotube. Since this device is based on optically actuated micro-gripper, we also can investigate cell's properties while grabbing. Also these devices will be used to measure electrical properties of live cell. Further, optically actuated silicon based nano-electrode and nano-gripper will be tried to fabricate by using MEMS technology. 


\section{REFERENCE}

1. Kim, S., F. Shafiei, D. Ratchford, and X.Q. Li, Controlled AFM manipulation of small nanoparticles and assembly of hybrid nanostructures. Nanotechnology, 2011. 22(11).

2. Sugimoto, Y., P. Pou, O. Custance, P. Jelinek, M. Abe, R. Perez, and S. Morita, Complex patterning by vertical interchange atom manipulation using atomic force microscopy. Science, 2008. 322(5900): p. 413-417.

3. Meyer, G., L. Bartels, and K.-H. Rieder, Atom manipulation with the STM: nanostructuring, tip functionalization, and femtochemistry. Computational Materials Science, 2001. 20(3-4): p. 443-450.

4. Kürpick, U. and B. Fricke, Manipulation of $\mathrm{Cu}$ adatoms on anisotropic $\mathrm{Cu}$ surfaces using scanning tunneling microscopy. Surface Science, 2000. 460(1-3): p. 1-11.

5. Hla, S.W., A. Kühnle, L. Bartels, G. Meyer, and K.H. Rieder, Controlled lateral manipulation of single diiodobenzene molecules on the Cu(111) surface with the tip of a scanning tunnelling microscope. Surface Science, 2000. 454-456: p. 10791084.

6. Gauthier, S., Atomic and molecular manipulations of individual adsorbates by STM. Applied Surface Science, 2000. 164(1-4): p. 84-90.

7. Zeppenfeld, P., C.P. Lutz, and D.M. Eigler, Manipulating atoms and molecules with a scanning tunneling microscope. Ultramicroscopy, 1992. 42-44(Part 1): p. 128-133.

8. Gao, H.J. and L. Gao, Scanning tunneling microscopy of functional nanostructures on solid surfaces: Manipulation, self-assembly, and applications. Progress in Surface Science. 85(1-4): p. 28-91.

9. Hertel, T., R. Martel, and P. Avouris, Manipulation of Individual Carbon Nanotubes and Their Interaction with Surfaces. The Journal of Physical Chemistry B, 1998. 102(6): p. 910-915.

10. Lee, J., S. Kwon, J. Choi, G. Kang, Y. Kwak, and S. Kim, Nanogripper using Carbon Nanotube, in NSTI-Nanotech 20042004.

11. Lee, J. and S. Kim, Manufacture of a nanotweezer using a length controlled CNT arm. Sensors and Actuators A: Physical, 2005. 120(1): p. 193-198. 
12. Watanabe, H., C. Manabe, T. Shigematsu, K. Shimotani, and M. Shimizu, Single molecule DNA device measured with triple-probe atomic force microscope. Applied Physics Letters, 2001. 79(15): p. 2462-2464.

13. Macdonald, N.C., L.Y. Chen, J.J. Yao, Z.L. Zhang, J.A. Mcmillan, and D.C. Thomas, Selective chemical vapor deposition of tungsten for microelectromechanical structures. Sensors and Actuators, 1989. 20: p. 123-133.

14. Yao, J.J. and N.C. Macdonald, Time-resolved scanning electron microscopy analysis of nanodynamical structures. Scanning Microscopy, 1992. 6: p. 937.

15. Shi, F., P. Ramesh, and S. Mukherjee, Simulation methods for micro-electromechanical structures (MEMS) with application to a microtweezer. Computers \& Structures, 1995. 56(5): p. 769-783.

16. Kolesar, E.S., M.D. Ruff, W.E. Odom, J.A. Jayachadran, J.B. McAllister, S.Y. Ko, J.T. Howard, P.B. Allen, J.M. Wilken, N.C. Boydston, J.E. Bosch, and R.J. Wilks, Single- and double-hot arm asymmetrical polysilicon surface micromachined electrothermal microactuators applied to realize a microengine. Thin Solid Films, 2002. 420-421: p. 530-538.

17. Hashiguchi, G. and H. Fujita. Micromachined nanoprobe and its application. in Sensors, 2002. Proceedings of IEEE. 2002.

18. Molhave, K. and O. Hansen, Electro-thermally actuated microgrippers with integrated force-feedback. Journal of Micromechanics and Microengineering, 2005. 15(6): p. 1265-1270.

19. Clevy, C., A. Hubert, J. Agnus, and N. Chaillet, A micromanipulation cell including a tool changer. Journal of Micromechanics and Microengineering, 2005. 15(10): p. S292-S301.

20. Chronis, N. and L.P. Lee, Electrothermally activated SU-8 microgripper for single cell manipulation in solution. Journal of Microelectromechanical Systems, 2005. 14(4): p. 857-863.

21. Zhou, J.W.L., H.Y. Chan, T.K.H. To, K.W.C. Lai, and W.J. Li, Polymer MEMS actuators for underwater micromanipulation. Ieee-Asme Transactions on Mechatronics, 2004. 9(2): p. 334-342.

22. Nguyen, N.T., S.S. Ho, and C.L.N. Low, A polymeric microgripper with integrated thermal actuators. Journal of Micromechanics and Microengineering, 2004. 14(7): p. 969-974.

23. Zubir, M.N.M., B. Shirinzadeh, and Y.L. Tian, A new design of piezoelectric driven compliant-based microgripper for micromanipulation. Mechanism and Machine Theory, 2009. 44(12): p. 2248-2264.

24. Zubir, M.N.M., B. Shirinzadeh, and Y.L. Tian, Development of a novel flexurebased microgripper for high precision micro-object manipulation. Sensors and Actuators a-Physical, 2009. 150(2): p. 257-266.

25. Zubir, M.N.M., B. Shirinzadeh, and Y.L. Tian, Development of novel hybrid flexure-based microgrippers for precision micro-object manipulation. Review of Scientific Instruments, 2009. 80(6).

26. Carbonari, R.C., E.C.N. Silva, and G.H. Paulino, Multi-actuated functionally graded piezoelectric micro-tools design: A multiphysics topology optimization 
approach. International Journal for Numerical Methods in Engineering, 2009. 77(3): p. 301-336.

27. Gotze, H. and L. Pagel, Development of a micro-manipulator based on piezoelectric-technology. Microelectronic Engineering, 2007. 84(5-8): p. 13331336.

28. Nah, S.K. and Z.W. Zhong, A microgripper using piezoelectric actuation for micro-object manipulation. Sensors and Actuators a-Physical, 2007. 133(1): $\mathrm{p}$. 218-224.

29. Jeon, C.S., J.S. Park, S.Y. Lee, and C.W. Moon, Fabrication and characteristics of out-of-plane piezoelectric micro grippers using MEMS processes. Thin Solid Films, 2007. 515(12): p. 4901-4904.

30. Perez, R., N. Chaillet, K. Domanski, P. Janus, and P. Grabiec, Fabrication, modeling and integration of a silicon technology force sensor in a piezoelectric micro-manipulator. Sensors and Actuators a-Physical, 2006. 128(2): p. 367-375.

31. Carrozza, M.C., A. Menciassi, G. Tiezzi, and P. Dario, The development of a LIGA-microfabricated gripper for micromanipulation tasks. Journal of Micromechanics and Microengineering, 1998. 8(2): p. 141-143.

32. Kohl, M., E. Just, W. Pfleging, and S. Miyazaki, SMA microgripper with integrated antagonism. Sensors and Actuators a-Physical, 2000. 83(1-3): p. 208213.

33. Menciassi, A., A. Moglia, S. Gorini, G. Pernorio, C. Stefanini, and P. Dario, Shape memory alloy clamping devices of a capsule for monitoring tasks in the gastrointestinal tract. Journal of Micromechanics and Microengineering, 2005. 15(11): p. 2045-2055.

34. Kim, P. and C.M. Lieber, Nanotube nanotweezers. Science, 1999. 286(5447): p. 2148-2150.

35. Akita, S., Y. Nakayama, S. Mizooka, Y. Takano, T. Okawa, Y. Miyatake, S. Yamanaka, M. Tsuji, and T. Nosaka, Nanotweezers consisting of carbon nanotubes operating in an atomic force microscope. Applied Physics Letters, 2001. 79(11): p. 1691-1693.

36. Cagliani, A., R. Wierzbicki, L. Occhipinti, D.H. Petersen, K.N. Dyvelkov, O.S. Sukas, B.G. Herstrom, T. Booth, and P. Boggild, Manipulation and in situ transmission electron microscope characterization of sub-100 $\mathrm{nm}$ nanostructures using a microfabricated nanogripper. Journal of Micromechanics and Microengineering, 2010. 20(3).

37. Jae Eun, J., C. Seung Nam, C. Youngjin, K. Dae Joon, D.G. Hasko, J. Jae Eun, K. Jong Min, and G.A.J. Amaratunga, A Nanogripper Employing Aligned Multiwall Carbon Nanotubes. Nanotechnology, IEEE Transactions on, 2008. 7(4): p. 389393.

38. Hashiguchi, G., T. Goda, M. Hosogi, K. Hirano, N. Kaji, Y. Baba, K. Kakushima, and H. Fujita, DNA Manipulation and Retrieval from an Aqueous Solution with Micromachined Nanotweezers. Analytical Chemistry, 2003. 75(17): p. 4347-4350.

39. Tsui, $\mathrm{K}$. and T. Cavanah, Using Xyvex Microgrippers TM Zyvex Nanomanipulators. 2008.

40. Beyeler, F., FT-Microgripper. 2007. 
41. Lu, S.X., Y. Liu, N. Shao, and B. Panchapakesan, Nanotube micro-optomechanical systems. Nanotechnology, 2007. 18(6).

42. Zhang, Y. and S. Iijima, Elastic Response of Carbon Nanotube Bundles to Visible Light. Physical Review Letters, 1999. 82(17): p. 3472.

43. Lu, S.X. and B. Panchapakesan, Nanotube micro-optomechanical actuators. Applied Physics Letters, 2006. 88(25).

44. Lu, S.X. and B. Panchapakesan, Optically driven nanotube actuators. Nanotechnology, 2005. 16(11): p. 2548-2554.

45. Cohn, R.W., M.M. Yazdanpanah, M. Hosseini, A. Safir, S. Pabba, S.A. Harfenist, F. Zamborini, and V.V. Dobrokhotov, Metallic nanostructure, self-assembly and testing method, 2008.

46. Dobrokhotov, V.V., M.M. Yazdanpanah, S. Pabba, A. Safir, and R.W. Cohn, Visual force sensing with flexible nanowire buckling springs. Nanotechnology, 2008. 19(3).

47. Yazdanpanah, M.M., Near room temperature self-assembly of nanostructure through Gallium reaction with metal thin films. 2006.

48. Yazdanpanah, M.M., S.A. Harfenist, A. Safir, and R.W. Cohn, Selective selfassembly at room temperature of individual freestanding $\mathrm{Ag}$ [sub 2] Ga alloy nanoneedles. Journal of Applied Physics, 2005. 98(7): p. 073510-7.

49. Yazdanpanah, M.M., M. Hosseini, S. Pabba, S.M. Berry, V.V. Dobrokhotov, A. Safir, R.S. Keynton, and R.W. Cohn, Micro-Wilhelmy and Related Liquid Property Measurements Using Constant-Diameter Nanoneedle-Tipped Atomic Force Microscope Probes. Langmuir, 2008. 24(23): p. 13753-13764.

50. Reifenberger, R., A. Raman, L. Biedermann, and M.M. Yazdanpanah, Ultra-soft atomic force microscope (USAFLM), 2009.

51. Safir, A., M.M. Yazdanpanah, S. Pabba, S.D. Cambron, F.P. Zamborini, R.S. Keynton, and R.W. Cohn. Fabrication of an insulated probe on a self-assembled metallic nanowire for electrochemical probing in cells. in Nanotechnology, 2006. IEEE-NANO 2006. Sixth IEEE Conference on. 2006.

52. Okamoto, H., ed. Desk Handbook: Phase Diagrams for Binary Alloys. Second ed. 2010, ASM International.

53. Yazdanpanah, M.M., 2010.

54. Biedermann, L.B., R.C. Tung, A. Raman, R.G. Reifenberger, M.M. Yazdanpanah, and R.W. Cohn, Characterization of silver-gallium nanowires for force and mass sensing applications. Nanotechnology, 2010. 21(30).

55. J, M.M., ed. Fundamentals of Microfabrication: The Science of Miniaturization. 2002, CRC Press: New York.

56. Easter, C. and C.B. O'Neal, Characterization of High-Pressure XeF2 VaporPhase Silicon Etching for MEMS Processing. Journal of Microelectromechanical Systems, 2009. 18(5): p. 1054-1061.

57. Sameoto, D., S.W. Lee, and M. Parameswaran. Wirebonding Characterization and Optimization on Thick Filmsu-8 MEMS Structures and Actuators. in SolidState Sensors, Actuators and Microsystems Conference, 2007. TRANSDUCERS 2007. International. 2007.

58. Harman, G.G., ed. Wire bonding in microelectronics. 2010. 
59. Johansson, A., J. Janting, P. Schultz, K. Hoppe, I.N. Hansen, and A. Boisen, SU-8 cantilever chip interconnection. Journal of Micromechanics and Microengineering, 2006. 16(2): p. 314-319.

60. Zhang, D., K. Ryu, X. Liu, E. Polikarpov, J. Ly, M.E. Tompson, and C. Zhou, Transparent, Conductive, and Flexible Carbon Nanotube Films and Their Application in Organic Light-Emitting Diodes. Nano Letters, 2006. 6(9): p. 18801886.

61. Shin, D.H., H.C. Shim, J.-W. Song, S. Kim, and C.-S. Han, Conductivity of films made from single-walled carbon nanotubes in terms of bundle diameter. Scripta Materialia, 2009. 60(8): p. 607-610.

62. Yu, X., R. Rajamani, K.A. Stelson, and T. Cui, Carbon nanotube based transparent conductive thin films. Journal of Nanoscience and Nanotechnology, 2006. 6(Compendex): p. 1939-1944.

63. $\mathrm{Hu}, \mathrm{L} ., \mathrm{D} . \mathrm{S}$. Hecht, and G. Grüner, Percolation in Transparent and Conducting Carbon Nanotube Networks. Nano Letters, 2004. 4(12): p. 2513-2517.

64. Birke, R.L., M.-H. Kim, and M. Strassfeld, Diagnosis of reversible, quasireversible, and irreversible electrode processes with differential pulse polarography. Analytical Chemistry, 1981. 53(6): p. 852-856.

65. Bockris, J.O.M., A.K.N. Reddy, and M. Gamboa-Aldeco, Modern electrochemistry: Fundamentals of electrodics. Vol. 2. 1998.

66. Myland, J.C. and K.B. Oldham, Cottrell's equation revisited: an intuitive, but unreliable, novel approach to the tracking of electrochemical diffusion. Electrochemistry Communications, 2004. 6(4): p. 344-350. 


\title{
CURRICULUM VITAE
}

\author{
PENG XU
}

\author{
EDUCATION: \\ 08/2009 - Present \\ (Expected) \\ University of Louisville, Louisville, USA \\ Master of Science in Mechanical Engineering \\ GPA: $4.00 / 4.00$ \\ $09 / 2005-07 / 2009$ \\ Hefei University of Technology, Hefei, China \\ Bachelor of Science in Applied Physics \\ GPA: 84/100 Rank: $10 / 99$
}

\section{PROFESSIONAL EXPERIENCE:}

08/2009_Present University of Louisville, Louisville, USA

Research Assistant, Small Systems Lab

09/2007-04/2008 Hefei University of Technology, Hefei, China

Research Assistant, Micro-electronic Laboratory

06/2007-10/2007 Institute of Plasma Physics Chinese Academy of Sciences, Hefei, China

SOCIAL ACTIVITIES:

10/2007-10/2008 Vice-President

Student Union of Anhui Province

President

Student Union of Hefei University of Technology

10/2006-10/2007 Vice-President 
Student Union of Hefei University of Technology

President

Student Union of Feicui Campus of HFUT

09/2006-09/2007

Editor-in-chief

The Book "Director of Freshmen"

\section{HONORS \& AWARDS:}

*Provincial Triple-A Student (2008)

*"Wang Zhenghu" Business Management Scholarship (2007)

*Excellent League Member (2007)

*Excellent Member of Student Union (2007)

* "Social Activity" Scholarship (2007)

*University First-class Scholarship (2006)

* Outstanding Student-Cadre of University (2006)

*Excellent Member of Summer Social Practice Team (2006) 